\title{
ARTICLE
}

\section{The KDM3A-KLF2-IRF4 axis maintains myeloma cell survival}

Hiroto Ohguchi ${ }^{1}$, Teru Hideshima ${ }^{1}$, Manoj K. Bhasin ${ }^{2}$, Gullu T. Gorgun ${ }^{1}$, Loredana Santo ${ }^{3}$, Michele Cea ${ }^{1} \dagger$, Mehmet K. Samur ${ }^{4}$, Naoya Mimura ${ }^{1}$, Rikio Suzuki ${ }^{1}$, Yu-Tzu Tai ${ }^{1}$, Ruben D. Carrasco ${ }^{1}$, Noopur Raje ${ }^{3}$, Paul G. Richardson ${ }^{1}$, Nikhil C. Munshi ${ }^{1,5}$, Hideo Harigae ${ }^{6}$, Takaomi Sanda ${ }^{7}$, Juro Sakai ${ }^{8}$ \& Kenneth C. Anderson ${ }^{1}$

KDM3A is implicated in tumorigenesis; however, its biological role in multiple myeloma $(\mathrm{MM})$ has not been elucidated. Here we identify KDM3A-KLF2-IRF4 axis dependence in $M M$. Knockdown of $K D M 3 A$ is toxic to $M M$ cells in vitro and in vivo. KDM3A maintains expression of KLF2 and IRF4 through H3K9 demethylation, and knockdown of KLF2 triggers apoptosis. Moreover, KLF2 directly activates IRF4 and IRF4 reciprocally upregulates KLF2, forming a positive autoregulatory circuit. The interaction of $M M$ cells with bone marrow milieu mediates survival of MM cells. Importantly, silencing of KDM3A, KLF2 or IRF4 both decreases $M M$ cell adhesion to bone marrow stromal cells and reduces $M M$ cell homing to the bone marrow, in association with decreased ITGB7 expression in MAF-translocated MM cell lines. Our results indicate that the KDM3A-KLF2-IRF4 pathway plays an essential role in MM cell survival and homing to the bone marrow, and therefore represents a therapeutic target.

\footnotetext{
${ }^{1}$ Department of Medical Oncology, Dana-Farber Cancer Institute, Boston, Massachusetts 02215, USA. 2 BIDMC Genomics, Proteomics, Bioinformatics and Systems Biology Center, Beth Israel Deaconess Medical Center, Boston, Massachusetts 02115, USA. ${ }^{3}$ MGH Cancer Center, Massachusetts General Hospital, Boston, Massachusetts 02114, USA. ${ }^{2}$ Department of Biostatistics and Computational Biology, Dana-Farber Cancer Institute, Boston, Massachusetts 02215, USA. ${ }^{5}$ West Roxbury Division, VA Boston Healthcare System, West Roxbury, MA 02132, USA. ${ }^{6}$ Department of Hematology and Rheumatology, Tohoku University Graduate School of Medicine, Sendai, Miyagi 980-8574, Japan. ${ }^{7}$ Cancer Science Institute of Singapore, Department of Medicine, National University of Singapore, Singapore 117599, Singapore. ${ }^{8}$ Division of Metabolic Medicine, Research Center for Advanced Science and Technology, University of Tokyo, Tokyo 153-8904, Japan. † Present address: Chair of Hematology, Department of Internal Medicine (DiMI), University of Genoa, IRCCS AOU San Martino-IST, Genoa 16100, Italy. Correspondence and requests for materials should be addressed to K.C.A. (email: kenneth_anderson@dfci.harvard.edu)
} 
H istone methylation contributes to the regulation of chromatin structure and function, thereby modulating gene expression, including transcription factors. Thus, histone methylation dynamics are critical for numerous biological processes including cell cycle regulation, DNA damage and stress response, as well as development and differentiation ${ }^{1,2}$. Histone methylation is tightly regulated by methyltransferases and demethylases that mediate the addition and removal of this modification, and, importantly, dysregulation of histone methylation is implicated in the pathogenesis of cancers, including multiple myeloma $(\mathrm{MM})^{3}$. For example, the $\mathrm{t}(4 ; 14)$ (p16;q32) is present in $15-20 \%$ of $M M$ patients, resulting in overexpression of WHSC1 (also known as MMSET/NSD2), a histone H3 lysine 36 (H3K36) methyltransferase. Depletion of WHSC1 expression induces apoptosis in MM cells with $\mathrm{t}(4 ; 14)$ translocation ${ }^{4}$, whereas catalytically active WHSC1 promotes oncogenic transformation in an H3K36 dimethylation-dependent manner 5 . In addition, $\sim 10 \%$ of MM patients without the $t(4 ; 14)$ translocation have inactivating somatic mutations in KDM6A (also known as UTX), an H3K27 demethylase ${ }^{6}$. Thus, the role of

a
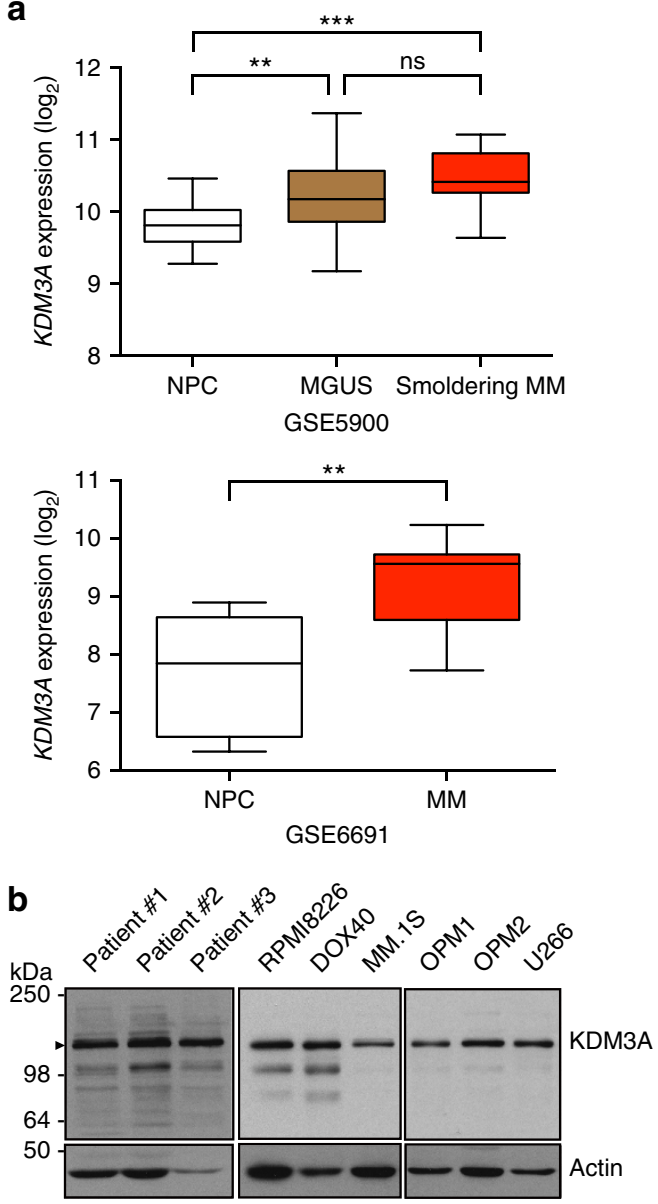

Figure 1 | KDM3A expression in $\mathbf{M M}$ cells. (a) KDM3A mRNA expression in patient MM samples. Publicly available microarray data sets (GSE5900 and GSE6691) were analysed for mRNA expression of KDM3A in normal plasma cells (NPC), monoclonal gammopathy of undetermined significance (MGUS), smoldering multiple myeloma (Smoldering MM) and MM cells. ${ }^{\star \star} P<0.01,{ }^{\star \star \star} P<0.001$. ns, not significant; analysis of variance (ANOVA) followed by Tukey's test. (b) Immunoblot analysis of KDM3A in patient MM cells and MM cell lines. Arrowhead represents KDM3A. Data are representative of at least two independent experiments, except for patient samples' blot. histone methylation modifiers in myelomagenesis is of particular interest.

KDM3A (also known as JMJD1A), a member of the Jumonji C-domain-containing histone demethylases, catalyses removal of $\mathrm{H} 3 \mathrm{~K} 9$ mono- and dimethylation (H3K9me1 and $\mathrm{H} 3 \mathrm{~K} 9 \mathrm{me} 2)^{7}$ and functions as a coactivator for androgen receptor. It is also a crucial regulator of spermatogenesis, embryonic stem cell selfrenewal, metabolic gene expression and sex determination ${ }^{7-12}$. Moreover, recent studies have implicated KDM3A in cancers $^{13-17}$. However, little is currently known about the biological role of KDM3A in the pathogenesis of MM.

KLF2, a nuclear DNA-binding transcription factor of the Krüppel zinc-finger family, is involved in various biological processes including blood vessel and lung formation, as well as T-cell homeostasis ${ }^{18}$. KLF2 is also expressed in B cells ${ }^{19,20}$, and absence of KLF2 in B-cell compartment causes abnormalities in B-cell subset identity, cellular trafficking and humoral immunity ${ }^{21-24}$. Thus, KLF2 plays a crucial role in maintaining homeostasis of B cells and plasma cells; however, the functional significance of KLF2 in MM remains to be defined.

IRF4, a member of the interferon regulatory family of transcription factors, is highly expressed in B cells and plasma cells, and has an essential role in controlling B cell to plasma cell differentiation and immunoglobulin class switching ${ }^{25-27}$. Furthermore, IRF4 is involved in myelomagenesis. Specifically, a subset of $\mathrm{MM}$ harbours $\mathrm{t}(6 ; 14)(\mathrm{p} 25 ; \mathrm{q} 32)$, which results in overexpression of IRF4 (ref. 28). Moreover, not only this subset with translocation of IRF4 but also all other subtypes of MM are dependent on IRF4 (ref. 29).

Here we investigate the biological significance of KDM3A in MM pathogenesis. We show that knockdown of KDM3A leads to apoptosis in MM cells, and that KDM3A directly upregulates KLF2 and IRF4 expression by removing H3K9 methyl marks at their promoters. We further show that knockdown of KLF2 induces apoptosis, and that KLF2 directly transactivates IRF4 promoter. Interestingly, KLF2 is also a direct target of IRF4, forming a positive autoregulatory loop in MM cells. In addition, we demonstrate that silencing of KDM $3 A, K L F 2$ or IRF4 impairs MM cell homing to the bone marrow. These findings suggest that the KDM3A-KLF2-IRF4 axis plays an essential role in MM cell growth and homing to the bone marrow, and therefore represents a potential therapeutic target.

\section{Results}

KDM3A is indispensable for MM cell survival. We first evaluated expression of $K D M 3 A$ mRNA in MM patient samples using publicly available gene expression profiling data because this jumonji demethylase has been implicated in the pathogenesis of several other cancers ${ }^{13-17}$. In two independent data sets ${ }^{30,31}$, KDM3A expression was significantly elevated in monoclonal gammopathy of undetermined significance and MM patient samples compared with normal plasma cells (Fig. 1a). We next examined KDM3A protein expression in MM cells. KDM3A protein was detected by immunoblotting in three patient $\mathrm{MM}$ cells and six human MM cell lines tested (Fig. 1b). This signal was increased by overexpression of KDM3A (Supplementary Fig. 1) and decreased by silencing of $K D M 3 A$ (Fig. 2a), confirming specific detection of KDM3A protein. Hence, we hypothesized that KDM3A may also play a role in the pathogenesis of MM.

To evaluate the functional role of KDM3A, MM cell lines were transduced with short hairpin RNAs (shRNAs) targeting KDM $3 A$ (shKDM3A \#1 and \#2) or control shRNA targeting luciferase (shLuc) by lentivirus. Transduction of KDM $3 A$-specific shRNAs reduced mRNA and protein levels of KDM3A in RPMI8226 and 
a
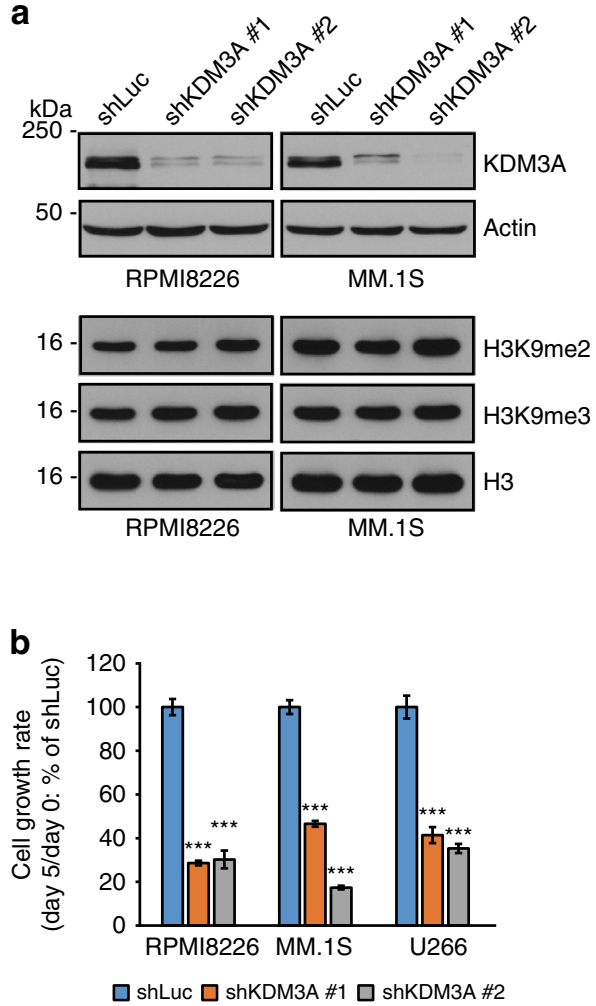

C

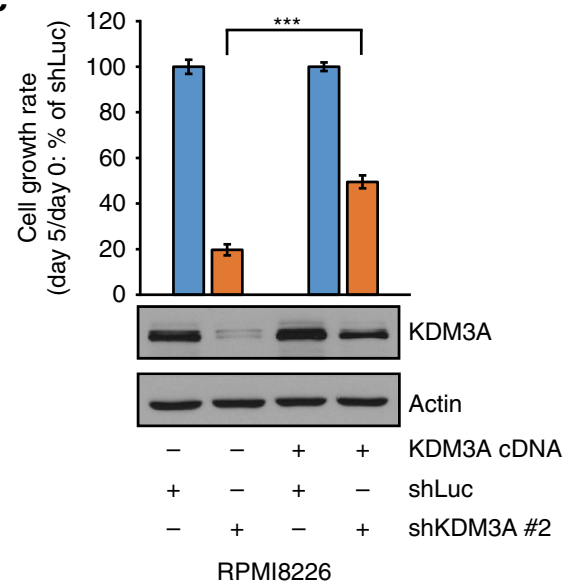

d

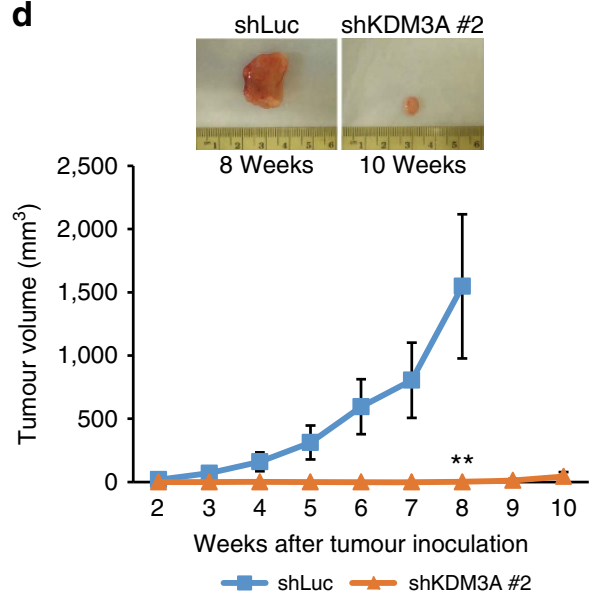

e
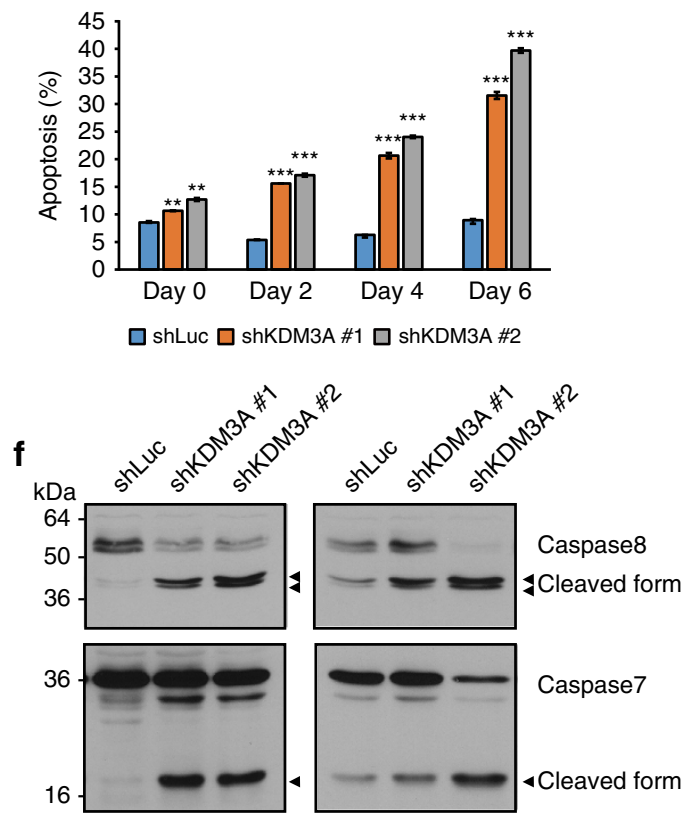

Caspase7

4 Cleaved form
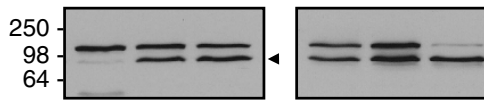

PARP

-Cleaved form
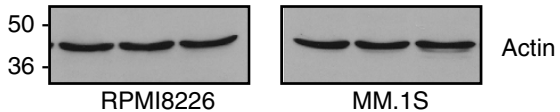

Figure 2 | KDM3A is indispensable for the survival of MM cells. (a) Immunoblot analysis of KDM3A, H3K9me2 and H3K9me3 in KDM3A-knockdown RPMI8226 and MM.1S cells. Cells were transduced with either KDM3A-specific shRNA (shKDM3A \#1 and \#2) or shLuc by lentivirus. Whole-cell lysates were subjected to immunoblot analysis. Actin or H3 served as the loading control for each membrane. (b) MM cell lines (RPMI8226, MM.1S and U266) were transduced with shKDM3A or control shLuc. Three days post infection, which was designated as day 0, cells were plated in 96-well plates. Cell viability was measured on days 0 and 5 by MTT assay, and the cell growth rate (day 5/day 0 ) relative to shLuc was determined. (c) RPMI8226 cells were retrovirally transduced with the KDM3A cDNA carrying synonymous mutations in the shKDM3A \#2 target sequence or with empty vector. Cells stably expressing the KDM3A cDNA or empty vector were then lentivirally transduced with shKDM3A \#2 or shLuc. The cell growth rate (day $5 /$ day 0 ) after lentiviral infection was determined for shKDM3A relative to shLuc. The growth rate for control shLuc in each cell type expressing the KDM3A cDNA or empty vector is set as $100 \%$. (d) MM.1S cells transduced with shKDM3A \#2 or shLuc ( $4 \times 10^{6}$ viable cells) were subcutaneously injected into SCID mice. Data represent mean \pm s.e.m. $n=7$ mice per group. An image of a representative tumour in each group is shown (top panel). (e) Apo2.7 immunostaining in RPMI8226 cells after knockdown of KDM3A. Cells were analysed 3 days post infection (day 0 ) and on days 2, 4, 6 for apoptosis by measuring apo2.7-positive cells. (f) Immunoblot analysis of caspase 8, caspase 7 and PARP in whole-cell lysates from KDM3A-knockdown RPMI8226 and MM.1S cells. Arrowheads indicate cleaved form. Actin served as the loading control. For $\mathbf{b}, \mathbf{c}, \mathbf{e}$, data represent mean \pm s.d. of quintuplicate cultures (b,c) or duplicate cultures (e). For $\mathbf{a}-\mathbf{c}, \mathbf{e}, \mathbf{f}$, data are representative of at least two independent experiments. ${ }^{\star \star} P<0.01,{ }^{\star \star \star} P<0.001$ compared with control; Student's $t$-test.

MM.1S cells (Fig. 2a and Supplementary Fig. 2a). Although KDM3A is a demethylase specific to H3K9me1 and me2 (ref. 7), the global H3K9me2 level was not significantly increased in MM cells transduced with $K D M 3 A$-specific shRNAs, similar to short interfering RNA-mediated KDM3A knockdown in HeLa cells ${ }^{32}$ (Fig. 2a). Importantly, knockdown of KDM3A significantly 
inhibited MM cell growth (Fig. 2b and Supplementary Fig. 2b), which was partially rescued by expression of the KDM $3 A$ cDNA carrying silent mutations in the shKDM3A-targeting sequence (Fig. 2c). Consistent with cell growth inhibition, DNA synthesis was also significantly reduced in $\mathrm{MM}$ cells transduced with shRNA targeting KDM3A versus control shRNA (Supplementary Fig. 2c). To further assess the effect of $K D M 3 A$ knockdown on MM cell growth in vivo, we subcutaneously injected MM.1S cells transduced with shRNA against KDM3A or shLuc into severe combined immunodeficient (SCID) mice. As shown in Fig. 2d, cell growth was significantly reduced in shKDM3A-treated MM.1S cells compared with shLuc-treated cells.

We next examined the molecular mechanism of cell growth inhibition. Quantitative analysis of apoptosis with flow cytometry using apo2.7 staining showed that apoptotic cells were significantly increased in KDM3A-knockdown RPMI8226 cells (Fig. 2e). Furthermore, immunoblot analysis revealed increased cleavage of caspase-8, -7 and poly (ADP-ribose) polymerase (PARP) after knockdown of KDM3A in RPMI8226, MM.1S and U266 cells (Fig. 2f and Supplementary Fig. 2d). Silencing of $K D M 3 A$ had little effect on the cell cycle profile (Supplementary Fig. 2e). These results suggest that knockdown of KDM $3 A$ triggers MM cell cytotoxicity via apoptosis.

KDM3A activates KLF2 and IRF4 through H3K9 demethylation. To identify the downstream effector targets of KDM3A, we next examined gene expression profiles after knockdown of KDM3A in RPMI8226 cells. With a cutoff of $\geq 1.5$-fold downregulation in $K D M 3 A$-knockdown cells relative to control cells, a total of 305 probe sets were downregulated in KDM3A-knockdown cells (Fig. 3a and Supplementary Data 1). The list includes genes encoding anti-apoptotic protein BCL2 (ref. 33), essential receptor for plasma cell survival TNFRSF17 (also known as BCMA) ${ }^{34,35}$, $B R A F^{36,37}$ and $F Y N^{38}$ (Fig. 3a). Among these putative KDM3A' targets, a gene of particular interest is KLF2, a key molecule in maintenance of $\mathrm{B}$ cell and plasma cell phenotype and function $^{21-24}$. Another relevant gene is IRF4, given its known crucial role in $\mathrm{MM}$ cell survival ${ }^{29}$.

To validate the microarray results, we measured $K L F 2$ and IRF4 mRNA using quantitative real-time PCR (QRT-PCR) and confirmed that expression of KLF2 and IRF4 was significantly reduced in KDM3A-silenced RPMI8226 cells (Fig. 3b). Immunoblot analysis further confirmed that protein levels of KLF2 and IRF4 were consistently diminished after knockdown of KDM3A (Fig. 3c,d). We identified a band at $\sim 40 \mathrm{kDa}$ as KLF2 protein, since this signal was increased by overexpression of KLF2 (Supplementary Fig. 3a, right panel) and decreased by silencing of KLF2 (Supplementary Fig. 3b, right panel), reflecting mRNA levels (Supplementary Fig. 3a,b, left panels). Knockdown of
KDM3A also decreased KLF2 and IRF4 expression at both the mRNA and protein levels in MM.1S and U266 MM cell lines (Fig. 3e,f).

To determine whether KDM3A directly regulates KLF2 and IRF4 expression, we next analysed KDM3A binding to KLF2 and IRF4 core promoters (near the transcriptional start sites) using chromatin immunoprecipitation (ChIP) assay in RPMI8226 cells. As a negative control we used the MYOD1 promoter region, since this cell line had no MYOD1 expression, and silencing of KDM $3 A$ had no effect on its expression. As shown in Fig. 3g, KDM3A bound to the promoters of KLF2 and IRF4, but not to the $M Y O D 1$ promoter. Moreover, knockdown of KDM3A abrogated KDM3A binding to KLF2 and IRF4 promoters, indicating that these are specific signals (Fig. $3 \mathrm{~h}$ ). We next evaluated $\mathrm{H} 3 \mathrm{~K} 9$ methylation levels on KLF2 and IRF4 promoters after silencing of KDM3A. H3K9me1 and me2 levels were significantly increased at both promoter regions in $K D M 3 A$-knockdown cells compared with control cells (Fig. 3i). Interestingly, we observed an increased trimethyl-H3K9 (H3K9me3) level at the KLF2 promoter, as was the case at Prm1 and Tnp1 promoters in the spermatids of $K D M 3 A$-deficient mice ${ }^{8}$, although $\mathrm{KDM} 3 \mathrm{~A}$ is unable to demethylate $\mathrm{H} 3 \mathrm{~K} 9 \mathrm{me} 3$ in vitro ${ }^{7}$. Taken together, these findings suggest that KDM3A directly regulates KLF2 and IRF4 expression by demethylating $\mathrm{H} 3 \mathrm{~K} 9$ methyl marks at their promoters in MM cells.

KLF2 is required for MM cell survival. We next confirmed that KLF2 protein was highly expressed in patient MM cells and MM cell lines (Fig. 4a). To investigate the biological significance of KLF2 in MM cells, shRNAs targeting KLF2 (shKLF2 \#1 and \#2) or shLuc were transduced into MM cells by lentivirus. We confirmed that the KLF2 expression level was decreased after knockdown of KLF2 using QRT-PCR and immunoblotting (Supplementary Fig. 3b). Importantly, silencing of KLF2 significantly reduced cell growth of MM cell lines (Fig. $4 \mathrm{~b}$ and Supplementary Fig. 4a), inhibited DNA synthesis (Supplementary Fig. 4b) as well as triggered apoptosis, evidenced by caspase-8, -7 and PARP cleavage (Fig. 4c). Expression of KLF2 cDNA carrying synonymous mutations in the shKLF2-targeting sequence partially rescued RPMI8226 cells from growth inhibition induced by shKLF2 (Fig. 4d). Significantly decreased in vivo growth of MM.1S cells transduced with shRNA against KLF2 compared with shLuc-transduced cells was noted in a subcutaneous xenograft model in SCID mice (Fig. 4e). Collectively, these data support the notion that KLF2 sustains MM cell survival.

IRF4 is a direct transcriptional target of KLF2 in MM cells. To identify the downstream targets of KLF2 in MM cells, we carried

Figure 3 | KDM3A directly regulates KLF2 and IRF4 expression through H3K9 demethylation at their promoters in MM cells. (a) Heatmap depicting the relative gene expression in RPMI8226 cells transduced with two independent shRNAs targeting KDM3A (shKDM3A \#1 and shKDM3A \#2) or shLuc. A total of 305 probes were selected based on $\geq 1.5$-fold downregulation in KDM3A-knockdown cells and clustered. (b-d) Quantitative real-time PCR (b) and immunoblot (c,d) analysis of KLF2 and IRF4 in RPMI8226 cells transduced with either shKDM3A or shLuc. After 3 days of infection, which is defined as day 0 in Fig. 2, cells were harvested for isolation of total RNA or whole-cell lysates. (b) Values represent the amount of mRNA relative to shLuc, defined as 1. (c) Arrowhead represents KLF2. (d) Signal intensity of each immunoblot was quantified using the ImageJ software. Results were normalized by Actin and are shown as relative signal intensity $($ shLuc $=1)$. Error bars represent s.e.m of three independent experiments. (e,f) Quantitative real-time PCR $(\mathbf{e})$ and immunoblot (f) analysis of KLF2 and IRF4 in MM.1S and U266 cells transduced with either shKDM3A or shLuc. After 3 days of infection, cells were harvested for isolation of total RNA or whole-cell lysates. (g) ChIP analysis showing KDM3A occupancy on KLF2 and IRF4 core promoters in RPMI8226 cells. Results were normalized to control IgG. The MYOD1 promoter region was used as negative control. (h) KDM3A occupancy is abrogated by KDM3A knockdown on KLF2 and IRF4 promoter regions in RPMI8226 cells. RPMI8226 cells transduced with either shKDM3A or shLuc were used for ChIP, followed by quantitative real-time PCR. (i) Enrichment of H3K9 methylation by KDM3A knockdown on KLF2 and IRF4 promoter regions in RPMI8226 cells. ChIP assays were performed with RPMI8226 cells transduced with either shKDM3A or shLuc. The relative enrichment over the input was assessed. Results are shown as fold enrichment compared with control shLuc. For $\mathbf{b}, \mathbf{e}, \mathbf{g}-\mathbf{i}$, error bars represent s.d. of triplicate measurements. For $\mathbf{b}-\mathbf{i}$, data are representative of at least two independent experiments. ${ }^{\star \star} P<0.01,{ }^{\star \star \star} P<0.001$ compared with control; Student's $t$-test. 
out microarray analysis after knockdown of KLF2 in RPMI8226 cells (Supplementary Data 2). Gene set enrichment analysis (GSEA) showed significant correlation between the genes downregulated by silencing of $K D M 3 A$ and $K L F 2$, indicating that KLF2 is a major downstream effector of KDM3A in MM cells
(Fig. 5a). Interestingly, we found that a gene encoding IRF4 was also significantly decreased after knockdown of KLF2 (Supplementary Data 2). To validate the KLF2 microarray data, we used QRT-PCR to show that IRF4 mRNA was significantly decreased in KLF2-silenced RPMI8226 cells (Fig. 5b). In a a

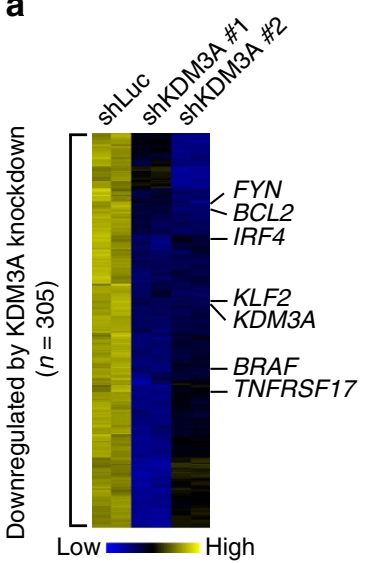

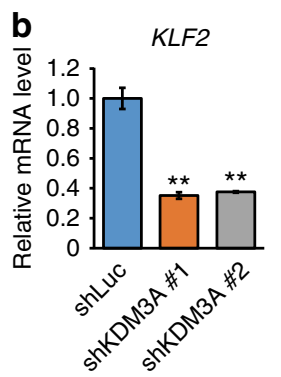

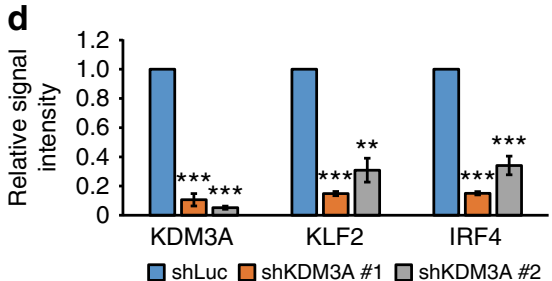

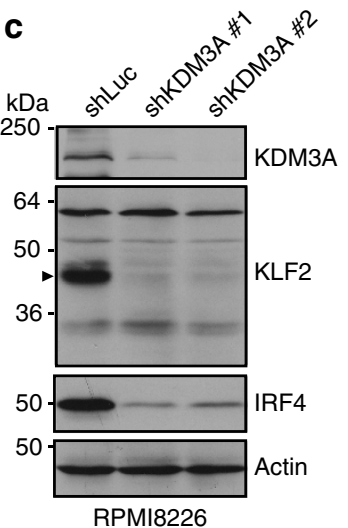
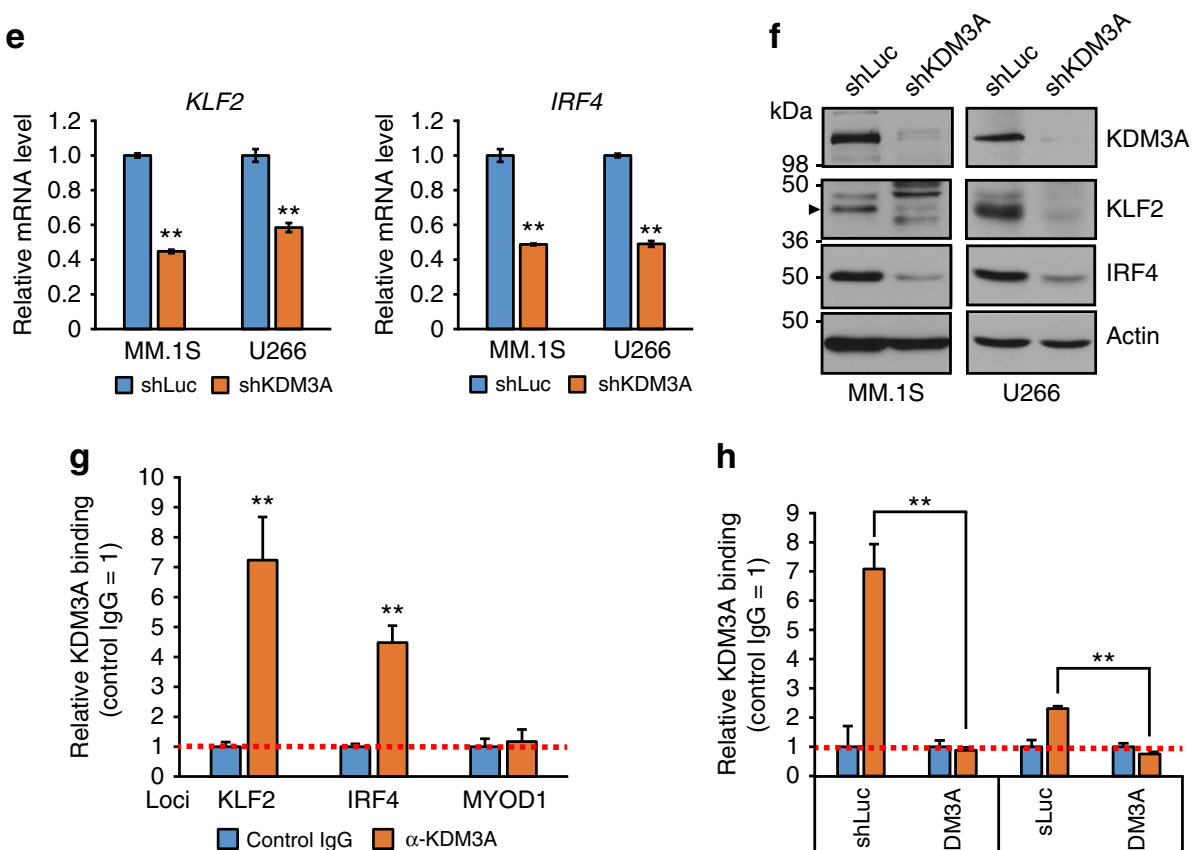

h
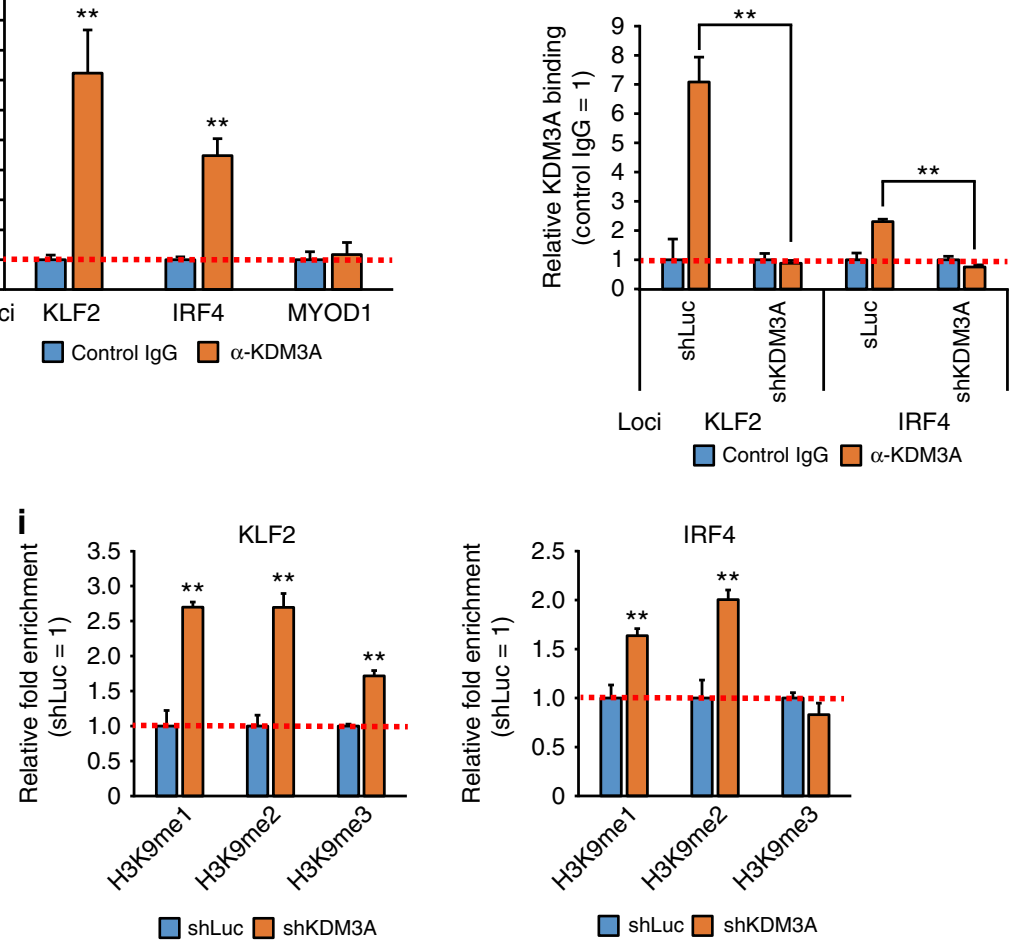

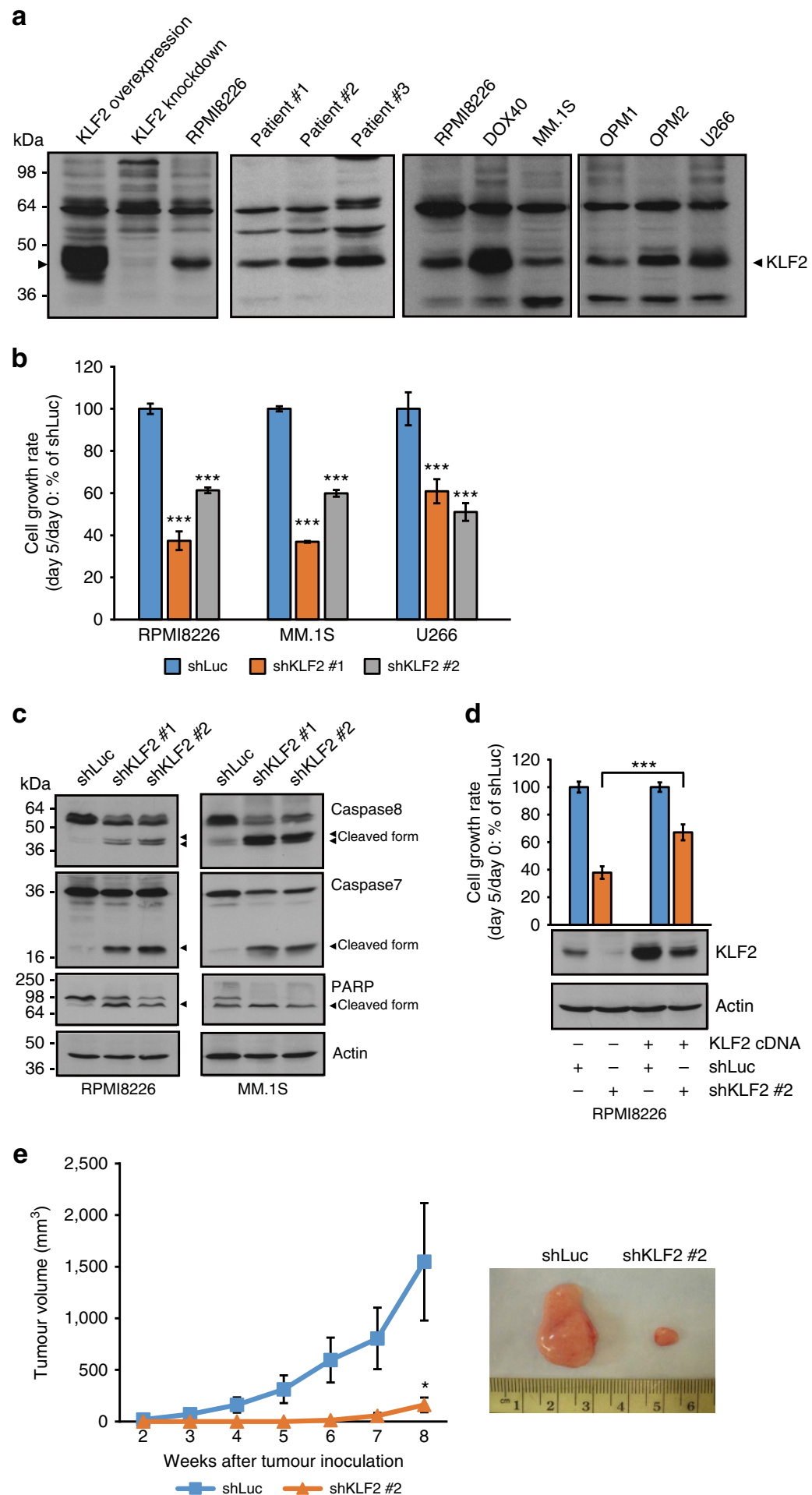

Figure 4 | KLF2 is required for MM cell survival. (a) Immunoblot analysis of KLF2 in patient MM cells and MM cell lines. Arrowhead represents KLF2. Whole-cell lysates from KLF2-overexpressed or knockdown RPMI8226 cells were used as positive or negative control for KLF2 antibody (N2212), respectively. (b) MM cell lines (RPMI8226, MM.1S and U266) were transduced with either KLF2-specific shRNA (shKLF2 \#1 and \#2) or control shLuc by lentivirus. Cell viability was measured on days 0 and 5 by MTT assay, and the cell growth rate (day $5 /$ day 0 ) relative to shLuc was determined. (c) Immunoblot analysis of caspase 8, caspase 7 and PARP in KLF2-knockdown RPMI8226 and MM.1S cells. Arrowheads indicate cleaved form. Actin served as the loading control. (d) RPMI8226 cells expressing the KLF2 cDNA carrying synonymous mutations in the shKLF2 \#2 target sequence or empty vector were transduced with shKLF2 \#2 or shLuc by lentivirus. The growth rate for control shLuc in each cell type expressing the KLF2 cDNA or empty vector is set as $100 \%$. (e) MM.1S cells transduced with shKLF2 \#2 or shLuc ( $4 \times 10^{6}$ viable cells) were subcutaneously injected into SCID mice. Data represent mean \pm s.e.m. $n=7$ mice per group. An image of a representative tumour in each group is shown (right panel). Data of control shLuc group are the same data shown in Fig. $2 \mathrm{~d}$ since subcutaneous xenograft studies of MM.1S cells treated with shKDM3A and shKLF2 were performed simultaneously with a common control shLuc group. For $\mathbf{b}, \mathbf{d}$, data represent mean \pm s.d. of quintuplicate cultures. For $\mathbf{a}-\mathbf{d}$, data are representative of at least two independent experiments, except for patient samples' blot (a). ${ }^{\star} P<0.05,{ }^{\star \star \star} P<0.001$ compared with control; Student's $t$-test. 
a

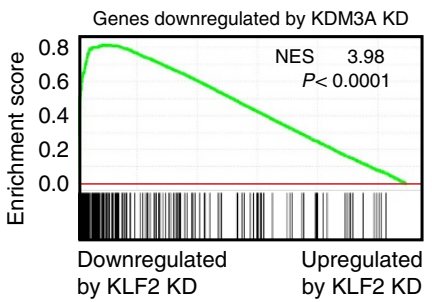

b
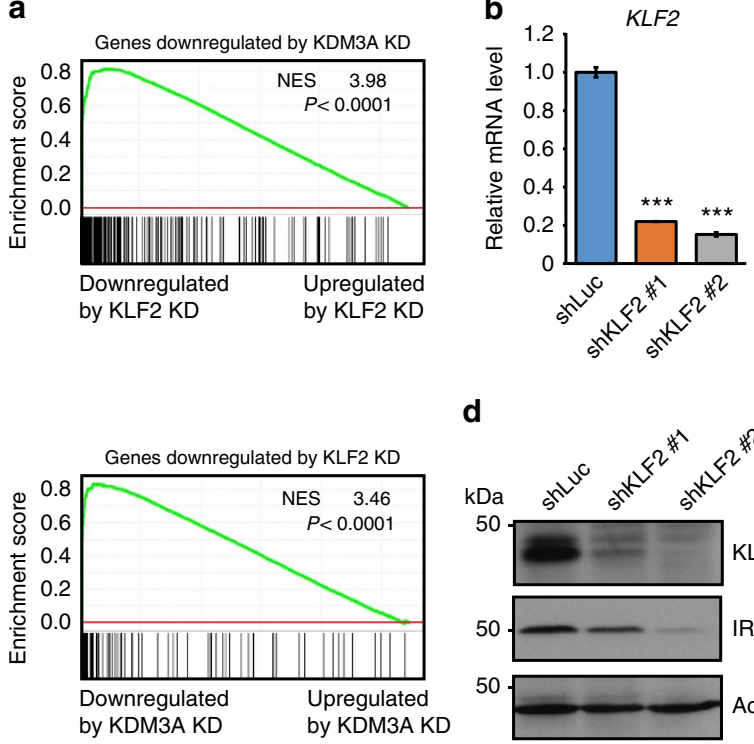

d

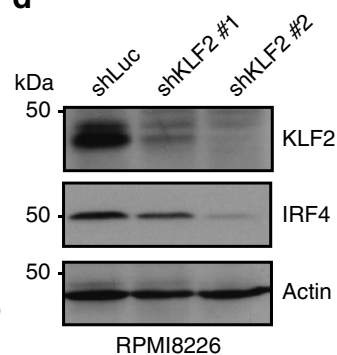

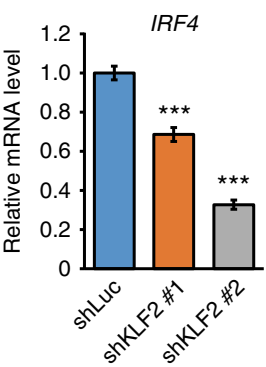

c
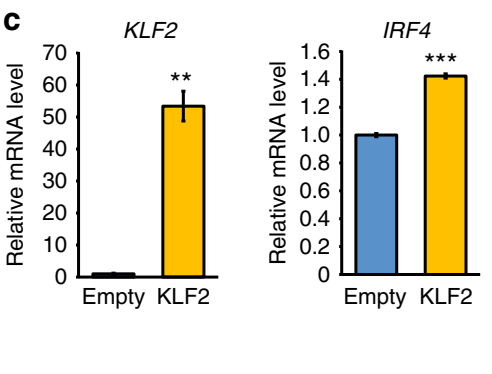

f
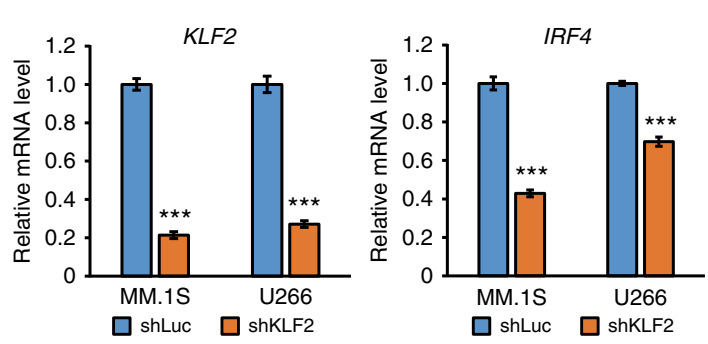

h

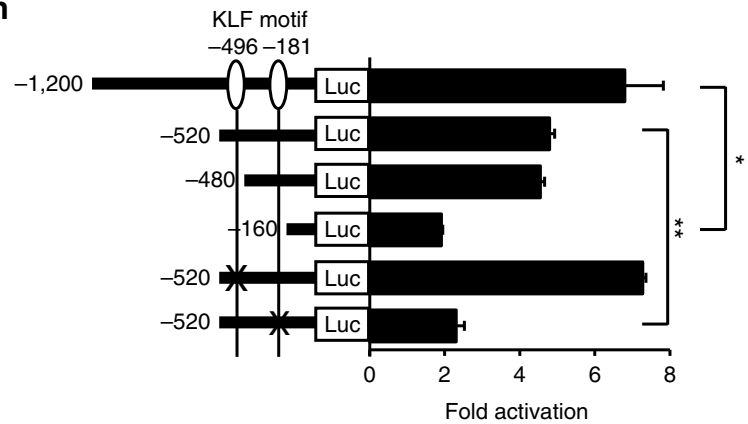

Inverted KLF motif(-496) $5^{\prime}$-GCCGAGGGTGCTCCC- $3^{\prime}$

KLF motif (-181) 5'-AACCC CACCCCGGCC-3'

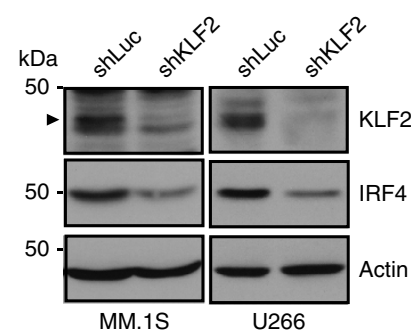

i

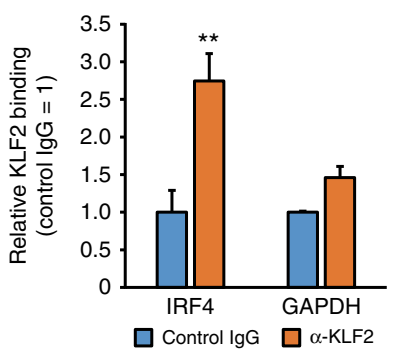

e

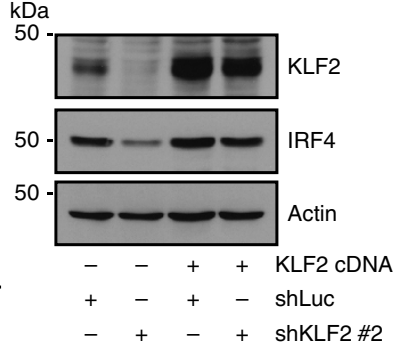

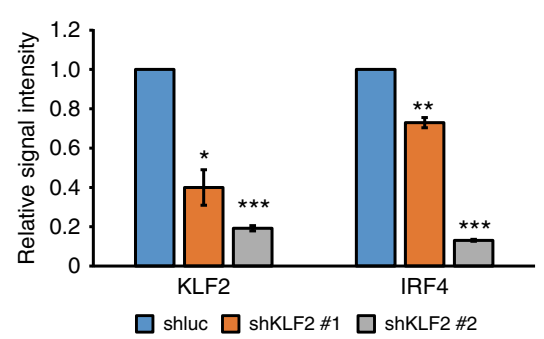

$\mathbf{g}$

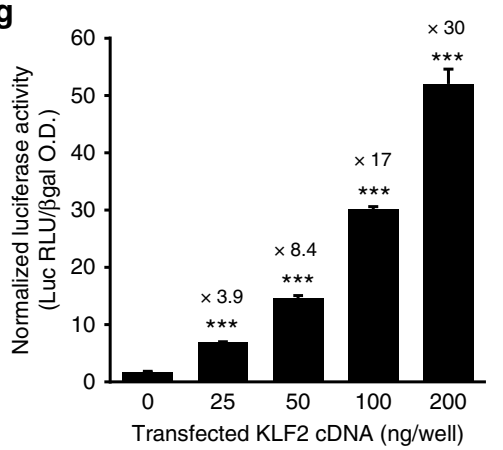

j

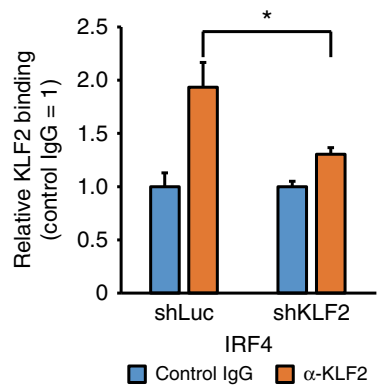

Figure 5 | IRF4 is a direct transcriptional target of KLF2 in MM cells. (a) Microarray analysis in RPMI8226 cells transduced with shKDM3A, shKLF2 or shLuc was performed. The genes significantly downregulated by KDM3A or KLF2 knockdown compared with control were used as gene sets for the GSEA. Normalized enrichment score (NES) and P values are shown. (b,c) Quantitative real-time PCR of KLF2 and IRF4 after knockdown (b) and overexpression (c) of KLF2 in RPMI8226 cells. (d) Immunoblot analysis of KLF2 and IRF4 after knockdown of KLF2 in RPMI8226 cells. Shown are the relative signal intensity $($ shLuc $=1)$ normalized by Actin. Error bars represent s.d. of two independent experiments. (e) RPMI8226 cells expressing the KLF2 cDNA carrying synonymous mutations in the shKLF2 \#2 target sequence or empty vector were transduced with shKLF2 \#2 or shLuc. Whole-cell lysates were subjected to immunoblot analysis. (f) Quantitative real-time PCR and immunoblot analysis of KLF2 and IRF4 after knockdown of KLF2 in MM.1S and U266 cells. (g) Transactivation of the IRF4 promoter by KLF2. The indicated amounts of KLF2 expression plasmids were transfected into 293 T cells together with the human IRF4 promoter-luciferase reporter. The value above each bar indicates the induction level compared with empty vector. (h) $293 \mathrm{~T}$ cells were co-transfected with the indicated IRF4-luciferase reporter and $0.04 \mu \mathrm{g}$ of KLF2 expression plasmid or empty vector, and then assayed for luciferase activity. The fold activation (normalized luciferase activity co-transfected with KLF2 expression plasmid relative to empty vector) is shown. (i) ChIP analysis showing KLF2 occupancy on IRF4 promoter in RPMI8226 cells. GAPDH promoter was used as negative control. (j) KLF2 occupancy is abrogated by KLF2 knockdown on IRF4 promoter in RPMI8226 cells. RPMI8226 cells transduced with either shKLF2 or shLuc were used for ChIP. For $\mathbf{b}, \mathbf{c}, \mathbf{f}, \mathbf{i}, \mathbf{j}, \mathbf{j}$, error bars represent s.d. of triplicate measurements. For $\mathbf{g}, \mathbf{h}$, data represent mean \pm s.d. of three $(\mathbf{g})$ or two $(\mathbf{h})$ biological replicates. For $\mathbf{b}-\mathbf{j}$, data are representative of at least two independent experiments. ${ }^{\star} P<0.05,{ }^{\star \star} P<0.01,{ }^{\star \star \star} P<0.001$ compared with control; Student's $t$-test. 
complementary manner, expression of IRF4 was increased in KLF2-overexpressed RPMI8226 cells (Fig. 5c). Consistent with the mRNA level, IRF4 protein expression was reduced after knockdown of KLF2 (Fig. 5d), and expression of KLF2 protein rescued IRF4 protein expression after knockdown of KLF2 (Fig. 5e). Depletion of KLF2 also resulted in decreased IRF4 expression at the mRNA and protein levels in MM.1S and U266 cells (Fig. 5f).

To identify the mechanisms regulating IRF4 expression, we next performed promoter reporter assays in 293T cells using reporter constructs containing a DNA fragment of the human IRF4 promoter (between bp $-1,200$ and +14 ). As shown in Fig. 5g, expression of KLF2 significantly increased luciferase expression in a dose-dependent manner, suggesting that KLF2 transactivates the IRF4 promoter. There are two KLF consensusbinding motifs within $1.2-\mathrm{kb}$ region of the IRF4 promoter (Fig. 5h). To examine whether both elements were required to mediate effects of KLF2, we introduced a series of $5^{\prime}$ deletions or mutations of KLF motifs into the promoter region. Deletion of the sequence from $-1,200$ to -480 had minimal effects on the response to ectopic expression of KLF2 (Fig. 5h). A further deletion to -160 almost abolished KLF2-mediated promoter activation. Furthermore, mutation of the proximal KLF motif significantly reduced the transactivation by KLF2, whereas mutation of the distal KLF motif did not affect the sensitivity to KLF2 expression. These results indicate that this proximal KLF site is at least one of the pivotal sites for transactivation of the IRF4 gene by KLF2. There remained weak KLF2-mediated promoter activation in the construct that has a deletion or a mutation of this proximal KLF motif. Although the IRF4 promoter within a 160-bp region does not contain KLF consensus-binding motif $\left(5^{\prime}\right.$-CACCC-3'), this region includes GC-rich sites. Sp1-like/KLF proteins have affinities for different GC-rich sites ${ }^{18}$; therefore, there may be another potential KLF2-binding site in this proximal promoter.

Finally, to assess KLF2 binding to the IRF4 promoter in MM cells, we carried out ChIP assays in RPMI8226 cells using antibody against KLF2 or control IgG, followed by QRT-PCR. As shown in Fig. 5i, endogenous KLF2 bound to the IRF4 proximal promoter. This ChIP signal was significantly reduced after knockdown of KLF2, indicating that this signal is KLF2-specific (Fig. 5j). Taken together, these data provide evidence that IRF4 is a direct target of KLF2 in MM cells.

KLF2 is a direct target of IRF4 transactivation in MM cells. Since transcription factors have been reported to form an autoregulatory feedback loop to sustain their expression in some models ${ }^{29,39,40}$, we hypothesized that IRF4 might regulate KLF2 expression. As expected, knockdown of IRF4 downregulated KLF2 expression at both the mRNA and protein levels in three MM cell lines (Fig. 6a,b). Furthermore, overexpression of IRF4 upregulated KLF2 expression (Fig. 6c), and expression of IRF4 protein partially rescued KLF2 protein expression after knockdown of IRF4 in RPMI8226 cells (Fig. 6d). We next ascertained whether IRF4 directly binds to the KLF2 locus. ChIP assays demonstrated that IRF4 bound to KLF2 second intron that contains tandem IRF4 motifs (GAAA), but not to the regions that harbour tandem IRF4 motifs, on the KLF2 promoter in RPMI8226 cells (Fig. 6e). These data indicate that KLF2 is a direct target of IRF4 in MM cells.

Collectively, these results suggest that KLF2 activates IRF4 expression and vice versa, forming a positive autoregulatory loop in MM cells. Consistent with these findings, the set of genes downregulated by IRF4 knockdown ${ }^{29}$ was significantly enriched for genes downregulated by KLF2 knockdown (Fig. 6f).
The KDM3A KLF2 IRF4 axis sustains MM cell adhesion and homing. The bone marrow microenvironment plays a crucial role in the pathogenesis of $\mathrm{MM}^{41,42}$. In this context, KLF2 has been reported to control homing of plasma cells to the bone marrow ${ }^{24}$. We therefore hypothesized that the KDM3A-KLF2-IRF4 axis might regulate adhesion and homing of MM cells to the bone marrow. To test this notion, we first evaluated the effect of silencing of KDM3A, KLF2 or IRF4 on MM cell adhesion and migration in vitro. As shown in Fig. 7a, knockdown of KDM3A, KLF2 or IRF4 decreased adhesion of RPMI8226, MM.1S and U266 cells to bone marrow stromal cells and fibronectin, without affecting the adhesion to bovine serum albumin (BSA), which served as a negative control. Knockdown of KDM3A, KLF2 or IRF4 also reduced MM.1S migratory ability towards CXCL12 in a transwell migration assay (Fig. 7b). We finally investigated the effect of silencing of KDM $3 A$, KLF2 or IRF4 on MM cell homing to the bone marrow in vivo. Quantitative analysis of the bone marrow homing of MM.1S-Luc-GFP cells was performed. An equal number of MM.1S-Luc-GFP cells stably expressing shRNA targeting KDM3A, KLF2 or IRF4 was injected via tail vein into sublethally irradiated nonobese diabetic (NOD)-SCID mice. Bone marrow cells of femurs were harvested $24 \mathrm{~h}$ after cell injection, and green fluorescent protein (GFP)-positive cells were detected using flow cytometry. The bone marrow homing of KDM $3 A$, KLF2 or IRF4-depleted MM.1S cells was significantly reduced compared with control cells expressing shRNA against red fluorescent protein (RFP) (Fig. 7c).

Previous studies have shown that reduced plasma cell homing to the bone marrow is accompanied by decreased ITGB7 expression in B-cell-specific KLF2 knockout mice $^{24}$, and that ITGB7 modulates MM cell adhesion and homing to the bone marrow $^{43,44}$. We therefore next hypothesized that the KDM3AKLF2-IRF4 axis might control expression of ITGB7 in MM cells. Of note, silencing of KDM $3 A, K L F 2$ or IRF4 downregulated expression of ITGB7 in RPMI8226 and MM.1S, which are MAF-translocated cell lines with high ITGB7 expression (Fig. 7d and Supplementary Fig. 5a). This reduced ITGB7 expression was restored by expression of each of these cDNAs in RPMI8226 cells, indicating that decreased ITGB7 expression after knockdown of KDM3A, KLF2 or IRF4 is not an off-target effect (Fig. 7e). In contrast, knockdown of KDM3A or KLF2 did not downregulate other MM cell-relevant adhesion molecules such as ITGB1, ITGA4, ITGA5, MUC1, SDC1, CD44, CD147 and ICAM1 (Supplementary Fig. 5b). We then evaluated MM cell adhesion after knockdown of ITGB7. Consistent with previous observations ${ }^{43,44}$, knockdown of ITGB7 reduced adhesion of RPMI8226 and MM.1S cells to bone marrow stromal cells and fibronectin (Fig. 7f). Taken together, these results suggest that the KDM3A-KLF2-IRF4 axis regulates ITGB7 expression in MAFtranslocated MM cells, and regulates, at least in part, MM cell adhesion and homing to the bone marrow.

\section{Discussion}

KDM3A has recently been implicated in the development of various solid tumours including colorectal cancer, sarcoma, bladder cancer and lung cancer ${ }^{13-17}$. The specific roles of $\mathrm{KDM} 3 \mathrm{~A}$ in the pathogenesis of cancers are now being delineated. For example, Cho et al. ${ }^{17}$ showed that KDM3A regulated the HOXA1 gene, which in turn suppressed CCND1 transcription, resulting in $\mathrm{G} 1 / \mathrm{S}$ arrest in cancer cells. In the current study, we demonstrate that silencing of $K D M 3 A$ induces apoptosis in MM cells. Unlike other cancers, knockdown of KDM3A does not affect HOXA1 expression or cell cycle distribution in MM cells (Supplementary Fig. 2e). Rather our current data demonstrate that KDM3A activates expression of 

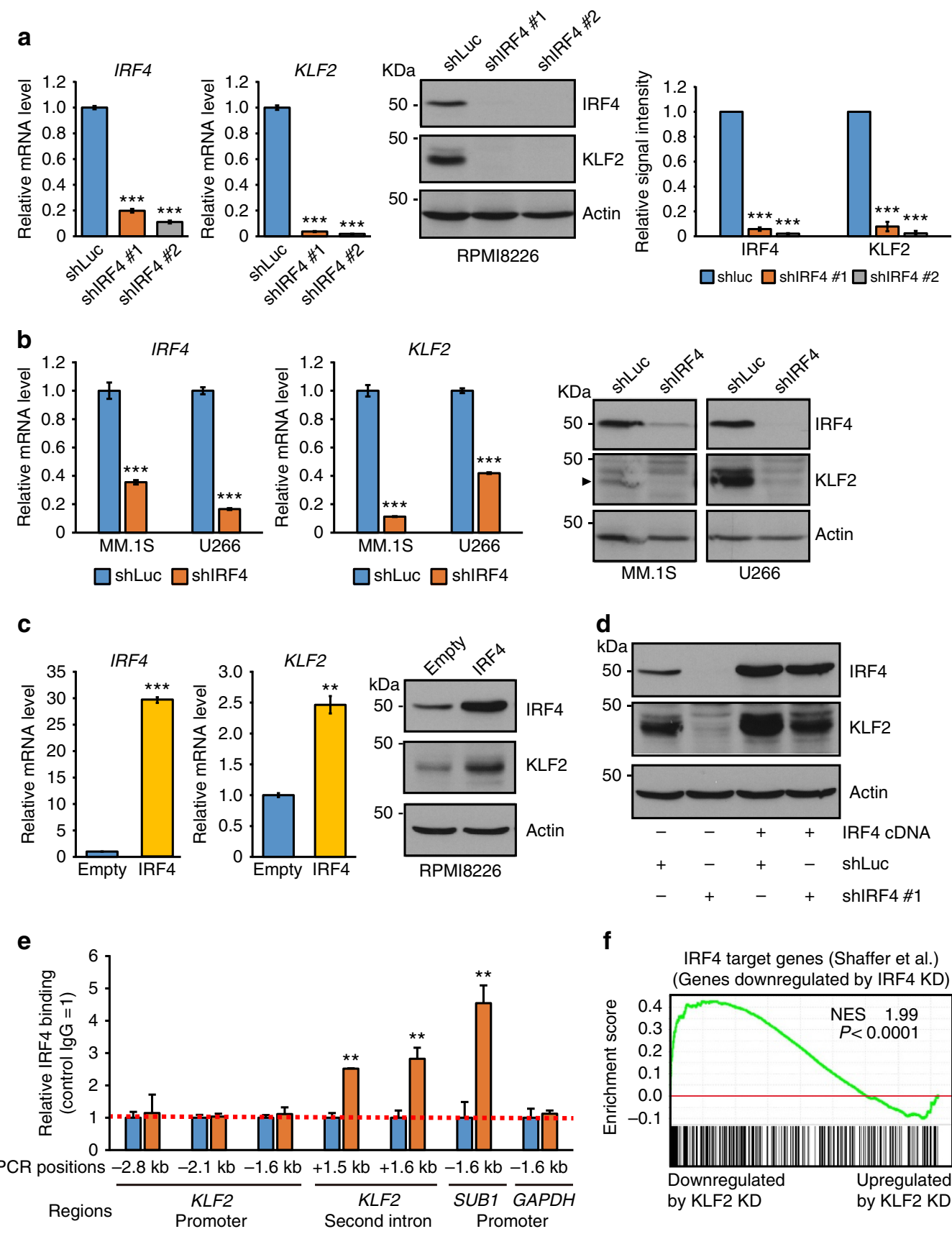

f

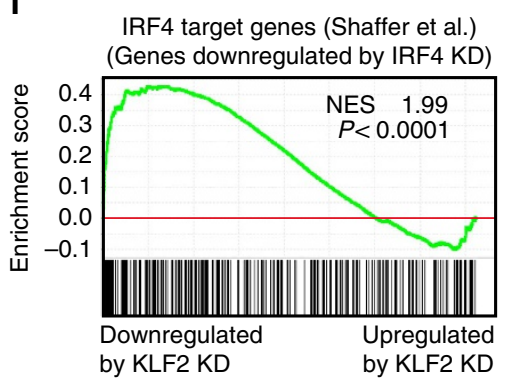

$\square$ Control IgG $\square \alpha-$ IRF4

Figure 6 | KLF2 is a direct target of IRF4 transactivation in MM cells. (a,b) Quantitative real-time PCR and immunoblot analysis of IRF4 and KLF2 in RPMI8226 (a), MM.1S and U266 (b) cells transduced with either shRNA targeting IRF4 (shIRF4) or shLuc. (a, right panel) Shown are the relative signal intensity (shLuc $=1$ ) normalized by Actin. Error bars represent s.e.m. of three independent experiments. (c) Quantitative real-time PCR and immunoblot analysis of IRF4 and KLF2 after overexpression of IRF4 in RPMI8226 cells. (d) RPMI8226 cells expressing the IRF4 cDNA or empty vector were transduced with shIRF4 \#1 that targets the $3^{\prime}$ untranslated region of IRF4 or shLuc by lentivirus. Whole-cell lysates were subjected to immunoblot analysis with antibodies against IRF4 and KLF2. (e) ChIP analysis showing IRF4 occupancy on KLF2 second intron in RPMI8226 cells. ChIP assays were performed for the regions that contain tandem IRF4 motifs on KLF2 promoter and intron (indicated PCR positions are relative to the transcriptional start site). Results were normalized to control lgG. The SUB1 promoter ${ }^{29}$ or GAPDH promoter was used as positive or negative control, respectively. (f) Significant correlation between the genes downregulated by KLF2 knockdown and IRF4 knockdown in MM cells. The genes significantly downregulated by IRF4 knockdown ${ }^{29}$ were used as gene set for GSEA. NES and $P$ values are shown. For $\mathbf{a}$ (left panel), b,c,e, error bars represent s.d. of triplicate measurements. For $\mathbf{a}-\mathbf{e}$, data are representative of at least two independent experiments. ${ }^{\star \star} P<0.01,{ }^{\star \star \star} P<0.001$ compared with control; Student's $t$-test.

KLF2 and IRF4 in MM cells. Depletion of IRF4 has been shown to induce $\mathrm{MM}$ cell death ${ }^{29}$. Our data also show that silencing of KLF2 triggers growth inhibition, indicating that not only IRF4, but also KLF2, are effector molecules in the KDM3A-dependent survival pathway. This notion is supported by GSEA, which shows that the list of genes downregulated by KDM3A silencing is significantly enriched for genes downregulated by KLF2 silencing. Nevertheless, overexpression of KLF2 or IRF4 did not rescue KDM3A knockdown-mediated cell growth inhibition (Supplementary Fig. 6a). In this study, we show that KDM3A 
sustains KLF2 and IRF4 expression through $\mathrm{H} 3 \mathrm{~K} 9$ demethylation at their promoters. Furthermore, KLF2 and IRF4 mutually transactivate expression of each other, generating a positive feedback loop in MM cells (Fig. 7g). These data suggest that not only each transcription factor but also KDM3A are indispensable for the expression of another transcription factor. In fact, overexpression of KLF2 did not rescue IRF4 expression in KDM3A-knockdown cells as well as overexpression of IRF4 did not rescue KLF2 expression (Supplementary Fig. 6b). These results suggest that overexpression of either KLF2 or IRF4 is not a
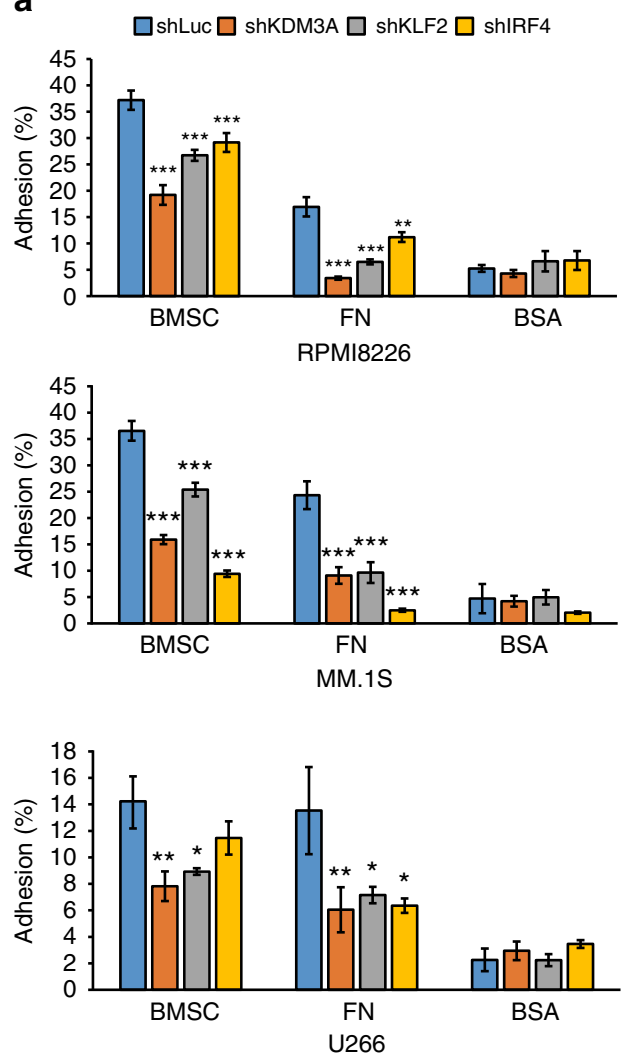

e

$\mathrm{KDa}$

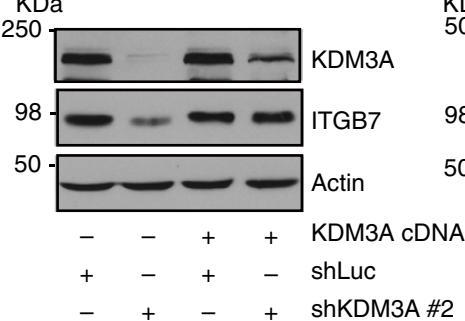

f
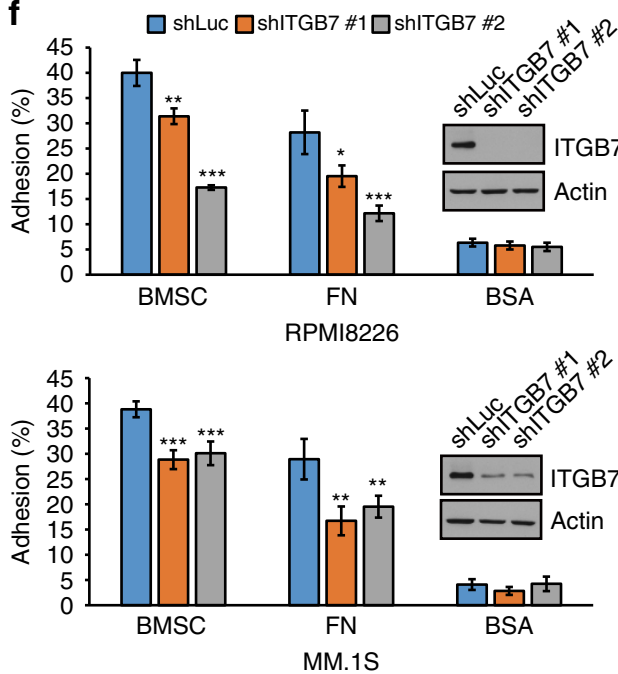

50 b
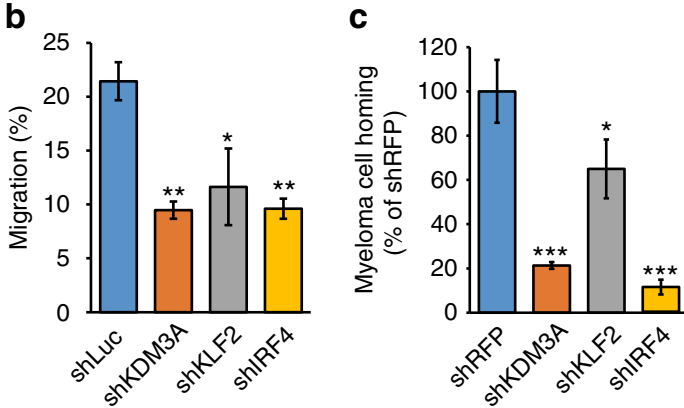

d
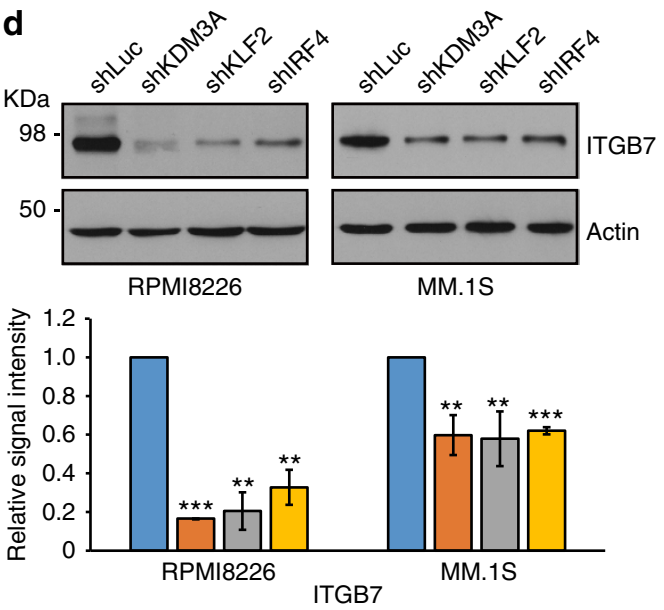

$\square$ shluc $\square$ shKDM3A $\square$ shKLF2 $\square$ shIRF4

$\mathrm{KDa}$

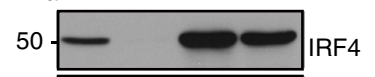

$98-\square$ ITGB7

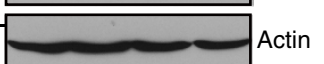

$98-\longrightarrow-\cdots$ ITGB7

50

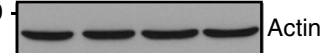

$-\quad+++$ IRF4 CDNA

+-+- shLuc

$+\quad-$ shLuc

$-+\quad+\quad+$ shIRF4 \#1

g
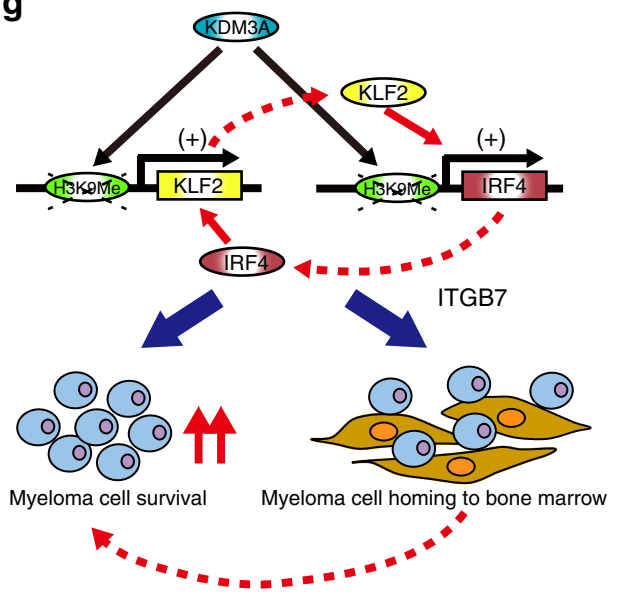
sufficient to rescue the KDM3A-knockdown phenotype, since both KLF2 and IRF4 are crucial in MM cell survival. KDM3A and these transcription factors may also cooperatively regulate the transcription machinery of common target genes critical for $\mathrm{MM}$ cell survival and therefore KLF2 or IRF4 may not be enough to rescue the effect of $K D M 3 A$ depletion, although further studies are required to address this issue. Thus, our data support the view that KDM3A maintains MM cell survival by coordinately regulating expression of essential transcription factors through histone demethylation. An interesting question is whether KDM3A contributes to the pathogenesis of other haematologic malignancies by the same mechanism, given its high expression at the same level as MM (Supplementary Fig. 7a). Interestingly, knockdown of $K D M 3 A$ also significantly impaired growth of diffuse large B-cell lymphoma (DLBCL) cell lines (Supplementary Fig. 7b). However, knockdown of KDM3A did not decrease KLF2 and IRF4 expression in DLBCL cell lines (Supplementary Fig. 7c), suggesting that KDM3A sustains survival of DLBCL via a different mechanism than that for MM. These data suggest a cancer-type-specific role of KDM3A.

The bone marrow microenvironment promotes MM cell survival ${ }^{41,42}$. Thus, adhesion and homing of MM cells to the bone marrow plays a key role in their growth and survival. Our study demonstrates that the KDM3A-KLF2-IRF4 pathway modulates $\mathrm{MM}$ cell adhesion and homing to the bone marrow, suggesting that this pathway maintains MM cell survival not only by preventing apoptosis but also by enhancing the interaction of MM cells with the bone marrow microenvironment (Fig. 7g). We found that knockdown of KDM3A, KLF2 or IRF4 downregulates ITGB7 expression in MAF-translocated MM cells. ITGB7 mediates MAF-driven MM cell adhesion to bone marrow stromal cells ${ }^{44}$, as well as $\mathrm{MM}$ cell homing to the bone marrow $^{43}$. ITGB7 may therefore contribute, at least in part, to the KDM3A-KLF2-IRF4 axis-dependent adhesion of MM cells to bone marrow stromal cells. Moreover, knockdown of KDM3A, KLF2 or IRF4 impairs adhesion of U266 MM cells, which only expressed low level of ITGB7, suggesting that this pathway mediates adhesion through not only ITGB7 but also via other unknown mechanisms.

Recent studies have described the genetic landscape of MM using whole-genome- and whole-exome sequencing, copy number array and cytogenetic analysis using samples from a large number of patients $36,45,46$. These studies identified mutated oncogenes including KRAS, NRAS and BRAF, as well as copy number gains of these oncogenes. One missense mutation in $K D M 3 A$ was found, but it was not recurrent and its functional significance is unknown ${ }^{45}$. Moreover, no copy number alterations or chromosomal rearrangements involving the $K D M 3 A$ locus were identified ${ }^{45,46}$. Nevertheless, MM cells are addicted to KDM3A. Thus, KDM3A addiction in myeloma may be 'nononcogene addiction,' a concept proposed by Luo et al. ${ }^{47}$ to describe the increased dependence of cancer cells on normal cellular activities of certain genes and pathways that are not inherently oncogenic by themselves. We found that KDM3A expression is elevated in patient MM cells relative to normal plasma cells (Fig. 1a). The MAF family of transcription factors, known driver oncogenes in $\mathrm{MM}^{44,48,49}$, may be associated with this aberrant KDM3A expression in a subset of MM, since $K D M 3 A$ expression is modestly but significantly elevated in the subgroup of newly diagnosed MM patients with MAF translocations and high MAF expression (Supplementary Fig. $8 \mathrm{a})^{50}$. The bone marrow microenvironment may also regulate KDM3A expression in MM cells with disease progression, since $K D M 3 A$ transcript levels in $M M$ cell lines were significantly upregulated after co-culture with bone marrow stromal cells (Supplementary Fig. 8b) ${ }^{42}$.

The present study demonstrates that knockdown of KDM3A induces apoptosis in MM cells. KDM3A-deficient mice exhibit spermatogenesis defects, male-to-female sex reversal and adult onset obesity, but no other additional phenotypes ${ }^{8,10-12}$. Hence, there may be a therapeutic window allowing for safely targeting KDM3A. A recent study revealed that KDM3A was distinct from other Jumonji C family members in both its structure and function. KDM3A forms a homodimer through its catalytic domains, and catalyses removal of $\mathrm{H} 3 \mathrm{~K} 9$ methylation via a two-step process in which two active sites of the dimer are crucial for the enzymatic activity ${ }^{51}$. This reaction mechanism is totally different from catalytic processes of other JumonjiC H3K9 demethylases such as PHF8 and KIAA1718, which neither form a homodimer nor catalyse successive two-step methylation ${ }^{52}$. These studies may provide the framework for developing small molecules that specifically inhibit KDM3A.

In conclusion, we identify $\mathrm{KDM} 3 \mathrm{~A}$ as a crucial epigenetic regulator of MM cell survival. We also show that KLF2 and IRF4 are downstream effectors in the KDM3A-dependent survival pathway, and that KLF2 and IRF4 regulate expression of each other. In addition, we demonstrate that this pathway also sustains MM cell adhesion and homing to the bone marrow, suggesting further supporting MM cell survival. Our data therefore suggest that modulation of histone $\mathrm{H} 3 \mathrm{~K} 9$ methylation by inhibiting KDM3A represents a promising novel therapeutic strategy in $\mathrm{MM}$, and provide the preclinical rationale for development of potent selective drugs targeting KDM3A for clinical evaluation in MM.

\footnotetext{
Figure 7 | The KDM3A-KLF2-IRF4 axis contributes to adhesion and homing of MM cells to the bone marrow. (a) RPMI8226, MM.1S and U266 cells expressing each shRNA were labelled with calcein-AM and incubated in bone marrow stromal cells (BMSC)- or fibronectin (FN)-coated plates. After washing plates, adherent cells were quantified using a fluorescence plate reader. Results are shown as per cent adhesion over the input. BSA-coated wells were used as negative control. (b) MM.1S cells transduced with each shRNA were placed in the upper chamber of a modified Boyden chamber and incubated with CXCL12 $(20 \mathrm{nM})$ added to the lower chamber. After $4 \mathrm{~h}$, cells that migrated to the lower chamber were quantified. Results are shown as per cent migration over the input. (c) MM.1S-Luc-GFP cells expressing each shRNA ( $15 \times 10^{6}$ viable cells) were intravenously injected into NOD-SCID mice. Twenty-four hours after injection, bone marrow cells of recipient mice were collected, and GFP-positive cells were detected using flow cytometry. Results are shown as homing efficacy relative to shRFP cells. Data represent mean \pm s.e.m. $n=4-5$ mice per group. ${ }^{\star} P<0.05,{ }^{\star \star \star} P<0.001$ compared with control; ANOVA followed by Dunnett's test. (d) Immunoblot analysis of ITGB7 after knockdown of KDM3A, KLF2, IRF4 in RPMI8226 and MM.1S cells. Shown are the relative signal intensity (shLuc $=1$ ) normalized by Actin. Error bars represent s.d. of two (RPMI8226) or three (MM.1S) independent experiments. (e) RPMI8226 cells expressing each cDNA were transduced with each shRNA as shown. Whole-cell lysates were subjected to immunoblot analysis. Data are representative of two independent experiments. (f) Adhesion assays were performed with RPMI8226 and MM.1S cells treated with either shITGB7 or shLuc. (g) Model of the KDM3A-KLF2-IRF4 axis in MM cells. KDM3A demethylates H3K9 of KLF2 and IRF4 promoter, resulting in upregulation of KLF2 and IRF4. KLF2 and IRF4 mutually activate expression of each other. The KDM3A-KLF2-IRF4 axis maintains MM cell survival and homing to the bone marrow. For $\mathbf{a}, \mathbf{b}, \mathbf{f}$, data represent mean \pm s.d. of quadruplicate incubations $(\mathbf{a}, \mathbf{f})$, or triplicate incubations $(\mathbf{b}) .{ }^{\star} P<0.05,{ }^{\star \star} P<0.01,{ }^{\star \star \star} P<0.001$ compared with control; Student's t-test.
} 


\section{Methods}

Cell culture. Human MM cell lines, RPMI8226, MM.1S and U266B1 (U266), were purchased from American Type Culture Collection (ATCC), and the identity of these cell lines was recently confirmed using STR profiling (Promega GenePrint 10 System). 293T packing cell line was also obtained from ATCC. MM.1S cell line expressing luciferase and GFP, MM.1S-Luc-GFP, was a kind gift from Dr Irene Ghobrial (Dana-Farber Cancer Institute, Boston, USA). Doxorubicin-resistant RPMI-DOX40 (DOX40) cell line was kindly provided by Dr William Dalton (Lee Moffitt Cancer Center, Tampa, USA). Human plasma cell leukaemia cell lines, OPM1 and OPM2, were kindly provided by Dr Edward Thompson (University of Texas Medical Branch, Galveston, USA). Human DLBCL cell lines, HBL-1 and SU-DHL-4, were kindly provided by Drs Bjoern Chapuy and Margaret A. Shipp (Dana-Farber Cancer Institute). Human DLBCL cell line KIS-1 (refs 53,54) was kindly provided by Dr Shinsuke Iida (Nagoya City University, Nagoya, Japan). All MM cell lines and KIS-1 were maintained in Roswell Park Memorial Institute (RPMI)-1640 medium containing $100 \mathrm{U} \mathrm{ml}^{-1}$ penicillin and $100 \mu \mathrm{g} \mathrm{ml}^{-1}$ streptomycin, supplemented with $10 \%(\mathrm{v} / \mathrm{v})$ fetal bovine serum and $2 \mu \mathrm{M}$ $\mathrm{L}$-glutamine, in $5 \% \mathrm{CO}_{2}$ at $37^{\circ} \mathrm{C}$. The growth media for SU-DHL-4 were further supplemented with $10 \mathrm{mM}$ HEPES. The growth media for HBL-1 were further supplemented with $10 \mathrm{mM}$ HEPES and $1 \mathrm{mM}$ sodium pyruvate. 293T cells were maintained in Dulbecco's Modified Eagle's Medium (DMEM) containing $100 \mathrm{U} \mathrm{ml}^{-1}$ penicillin and $100 \mu \mathrm{g} \mathrm{ml}^{-1}$ streptomycin, supplemented with $10 \%$ (v/v) fetal bovine serum.

Cell lines were tested to rule out mycoplasma contamination using the MycoAlert Mycoplasma Detection Kit (Lonza).

Primary MM cells and bone marrow stromal cells. Bone marrow samples were obtained from MM patients after informed consent and approval by the Institutional Review Board of the Dana-Farber Cancer Institute. Mononuclear cells were separated by Ficoll-Paque PLUS (GE Healthcare), and MM cells were purified by CD138-positive selection with anti-CD138 magnetic activated cell separation microbeads (Miltenyi Biotec). Long-term bone marrow stromal cells were established by culturing CD138-negative bone marrow mononuclear cells for 4-6 weeks in DMEM containing $100 \mathrm{U} \mathrm{ml}^{-1}$ penicillin and $100 \mu \mathrm{g} \mathrm{ml}^{-1}$ streptomycin, supplemented with $15 \%(\mathrm{v} / \mathrm{v})$ fetal bovine serum ${ }^{55}$.

Gene expression analysis using publicly available data sets. Gene Expression Omnibus data sets (accession numbers GSE5900 (ref. 30), GSE6691 (ref. 31), GSE36133 (ref. 56), GSE2658 (ref. 50) and GSE20540 (ref. 42)) were used for gene expression analysis. 212689_s_at is the probe for KDM3A transcript on Affymetrix Human Genome U133A Array or Human Genome U133 Plus 2.0 Array.

Immunoblot analysis. Cells were harvested, washed with PBS and lysed in RIPA buffer (Boston Bioproducts) containing a mixture of protease inhibitor (cOmplete, Mini, Roche) and $1 \mathrm{mM}$ phenylmethanesulfonyl fluoride (Sigma-Aldrich). The suspension was incubated for $30 \mathrm{~min}$ at $4{ }^{\circ} \mathrm{C}$ and centrifuged at top speed in a microfuge for $15 \mathrm{~min}$ at $4{ }^{\circ} \mathrm{C}$. The supernatant was used as whole-cell lysates. For immunoblotting of histone protein, cells were lysed in TOPEX buffer $(300 \mathrm{mM}$ $\mathrm{NaCl}, 50 \mathrm{mM}$ Tris-HCl, pH7.5, $1 \%$ SDS, $0.5 \%$ Triton X-100, $1 \mathrm{mM}$ dithiothreitol and 33.33 unit $\mathrm{ml}^{-1}$ Benzonase) with a mixture of protease inhibitor ${ }^{57}$, incubated for $30 \mathrm{~min}$ at $4{ }^{\circ} \mathrm{C}$ and these lysates were used as whole-cell lysates. Protein concentration was measured with the Bio-Rad Protein Assay (Bio-Rad). Samples were mixed with $4 \times$ Laemmli's SDS loading buffer (Boston Bioproducts), boiled at $95^{\circ} \mathrm{C}$ for $5 \mathrm{~min}$ and subjected to SDS-PAGE. After proteins were transferred to a nitrocellulose membrane (Bio-Rad), the membranes were blocked in Tris-buffered saline containing $0.1 \%(\mathrm{v} / \mathrm{v})$ Tween 20 and $5 \%(\mathrm{w} / \mathrm{v})$ nonfat dry milk for $1 \mathrm{~h}$ at room temperature. Immunoblots were carried out with the antibodies described in Supplementary Table 1, and visualized using the ECL Western Blotting Detection Reagents (GE Healthcare). For stripping off antibody, OneMinute Plus Western Blot Stripping Buffer (GM Biosciences) was used according to the manufacturer's instruction. Signal intensity was quantified using the ImageJ software (National Institutes of Health). Uncropped images of all blots are provided in Supplementary Fig. 9.

Expression plasmids. pLKO.1-based plasmids for shRNAs were obtained from the RNAi Consortium (Broad Institute). The RNAi Consortium clone ID and target sequence of each vector are provided in Supplementary Table 2. The human $K D M 3 A$ cDNA was amplified using PCR and ligated into the HpaI and XhoI sites of pMSCV retroviral expression vector (Clontech). The human KLF2 cDNA was amplified using PCR and ligated into pCR-Blunt (Invitrogen), and then cloned into the EcoRI site of pcDNA3 (Invitrogen) or pMSCV retroviral expression vector (Clontech). The human IRF4 CDNA was amplified using PCR and ligated into the EcoRI and XhoI sites of pMSCV retroviral expression vector (Clontech). To generate shRNA-resistant cDNAs, six point mutations (silent mutations) were introduced into shRNA-targeting sequences of KDM3A and KLF2 with the QuikChange II Site-Directed Mutagenesis kit (Agilent Technologies). Cloning and mutagenesis primers used in this study are shown in Supplementary Tables 3 and Supplementary Table 4.
Viral production and infection. On day 0, 293T packaging cells were plated at a density of $6 \times 10^{5}$ cells per six-well plates. On day 1 , cells were transfected with $500 \mathrm{ng}$ of pLKO.1 plasmid, $500 \mathrm{ng}$ of pCMV-dvpr and $100 \mathrm{ng}$ of VSV-G for lentivirus packaging, or were transfected with $500 \mathrm{ng}$ of pMSCVneo plasmid, $500 \mathrm{ng}$ of pMD-MLV and $100 \mathrm{ng}$ of VSV-G for retrovirus packaging, using TransIT-LT1 Transfection Reagent (Mirus), according to the manufacturer's instructions. On day 2, media were replaced and cells were cultured for an additional $24 \mathrm{~h}$ to obtain viral supernatants. On day 3 , media containing virus were harvested, passed through $0.45-\mu \mathrm{m}$ cellulose acetate membrane filters and used fresh for infection. Overall, $1 \times 10^{6}$ cells per $1 \mathrm{ml}$ of crude viral supernatants in the presence of $8 \mu \mathrm{g} \mathrm{ml}^{-1}$ polybrene were spinoculated at $800 \mathrm{~g}$ for $30 \mathrm{~min}$ at room temperature, and then incubated in $5 \% \mathrm{CO}_{2}$ at $37^{\circ} \mathrm{C}$ for $5 \mathrm{~h}$. Media were then replaced. After $24 \mathrm{~h}$ of viral infection, cells expressing shRNAs were selected with puromycin dihydrochloride (Sigma-Aldrich) at $1 \mu \mathrm{g} \mathrm{ml}^{-1}$ for 2 days, and then subjected to each assay. Cells expressing cDNAs were selected with G418 disulfate (Sigma-Aldrich) at $600 \mu \mathrm{g} \mathrm{ml}^{-1}$ for at least 7 days, and clones stably expressing cDNAs were subjected to rescue experiments. Puromycin and G418 concentrations were titrated to identify the minimum concentration of each drug that caused complete cell death of uninfected cells after 2 or 7 days, respectively.

RNA extraction and QRT-PCR. Total RNA was extracted using the RNeasy Mini Kit (Qiagen). cDNA was synthesized from $2 \mu \mathrm{g}$ of total RNA with oligo(dT) primers using the SuperScript III First-Strand Synthesis System (Invitrogen). Real-time PCR was performed in 96-well plates using the Applied Biosystems 7300 or 7500 Real-Time PCR System (Applied Biosystems). The PCR mixture contained $20 \mathrm{ng}$ of reverse-transcribed RNA, $200 \mathrm{nM}$ of forward and reverse primers and SYBR green PCR Master Mix (Applied Biosystems) in a final volume of $20 \mu \mathrm{l}$. The conditions were $95^{\circ} \mathrm{C}$ for $10 \mathrm{~min}$, followed by 40 cycles of $15 \mathrm{~s}$ at $95^{\circ} \mathrm{C}$ and $1 \mathrm{~min}$ at $60^{\circ} \mathrm{C}$. The relative amount of each transcript was calculated using the relative standard curve method. Cyclophilin A mRNA was used as the invariant control, and values were normalized by Cyclophilin A expression. Specific primers for each gene transcript are shown in Supplementary Table 5.

Cell growth assay. Cell growth was assessed by measuring 3-(4,5-Dimethyl-2thiazolyl)-2,5-diphenyl-2H-tetrazolium bromide (MTT, Sigma-Aldrich) dye absorbance ${ }^{58}$. Cells cultured in 96-well plates ( $100 \mu \mathrm{l}$ per well) were pulsed with $10 \mu \mathrm{l}$ of $5 \mathrm{mg} \mathrm{ml}^{-1}$ MTT for the last $4 \mathrm{~h}$ of cultures, followed by addition of $100 \mu \mathrm{l}$ of isopropanol containing $0.04 \mathrm{~N} \mathrm{HCl}$. Absorbance was measured at $570 \mathrm{~nm}$, with $630 \mathrm{~nm}$ as a reference wavelength, using a spectrophotometer (SpectraMax M3, Molecular Devices).

DNA synthesis was measured by ${ }^{3} \mathrm{H}$-thymidine (PerkinElmer) uptake $e^{58}$. Cells incubated in 96 -well plates were pulsed with ${ }^{3} \mathrm{H}$-thymidine $(0.25 \mathrm{Ci}$ per well) during the last $5 \mathrm{~h}$ of cultures. Cells were then harvested on fibreglass filters (Filtermat A, PerkinElmer) with Tomtec Cell Harvester, and ${ }^{3} \mathrm{H}$-thymidine incorporation was measured using 1450 MicroBeta TriLux scintillation counter (PerkinElmer).

Subcutaneous xenograft model. Nine-week-old male CB17 SCID mice (Charles River Laboratories) were used for this study. Overall, $4 \times 10^{6}$ viable MM.1S cells transduced with the corresponding shRNA (shKDM3A, shKLF2 or shLuc) were suspended in $100 \mu \mathrm{l}$ of PBS and then inoculated subcutaneously into the right flank of 200-cGy-irradiated mice. Tumour growth was monitored twice a week using an electronic caliper, and tumour volume was calculated using the formula: $\left(\right.$ length $\times$ width $\left.^{2}\right) \times 2^{-1}$, where length is greater than width. Animal studies were performed under a protocol approved by the Dana-Farber Institutional Animal Care and Use Committee.

Apoptosis assay. Quantification of apoptotic cells was performed by Apo2.7 immunostaining, which specifically detects the mitochondrial membrane antigen exposed on cells undergoing apoptosis ${ }^{59}$. Overall, $5 \times 10^{5}$ cells were incubated with $100 \mu \mathrm{g} \mathrm{ml}^{-1}$ of digitonin (Sigma-Aldrich) in $100 \mu \mathrm{l}$ of PBS with $2.5 \%$ (v/v) fetal bovine serum (PBSF) for $20 \mathrm{~min}$ at $4{ }^{\circ} \mathrm{C}$ to permeabilize cells. After washing with $2 \mathrm{ml}$ of PBSF, cells were incubated with $20 \mu \mathrm{l}$ of anti-Apo2.7-R-phycoerythrin (PE) or mouse $\mathrm{IgG}_{1}$ isotype control-PE (Beckmann Coulter) in $100 \mu \mathrm{l}$ of PBSF for $15 \mathrm{~min}$ at room temperature in the dark. After washing with $2 \mathrm{ml}$ of PBSF, cells were suspended with $1 \mathrm{ml}$ of PBSF and then analysed with flow cytometry (BD FACSCanto II, BD Biosciences). The proportion of apoptotic cells was assessed by measuring PE. In an optimization experiment with $\mathrm{IgG}_{1}$ isotype control, the possibility of nonspecific background was excluded.

Cell cycle assay. Cells were harvested and fixed with $70 \%$ ethanol for $20 \mathrm{~min}$ on ice. After washing with PBS twice, cells were incubated with $5 \mu \mathrm{g} \mathrm{ml}{ }^{-1}$ RNase (Roche) in PBS for $20 \mathrm{~min}$ at room temperature, and then resuspended in PBS containing $10 \mu \mathrm{g} \mathrm{ml}^{-1}$ propidium iodide (Sigma-Aldrich). The stained cells were analysed with flow cytometry (BD FACSCanto II, BD Biosciences), and the percentage of cells in G1, S and G2/M phases was determined using the ModFit LT software (Verity Software House). 
ChIP assay. ChIP assay was performed using the SimpleChIP Plus Enzymatic Chromatin IP Kit (Cell Signaling), according to the manufacturer's protocol. For ChIP with anti-KDM3A, $\sim 5 \times 10^{6}$ RPMI8226 cells were crosslinked with $1.5 \mathrm{mM}$ ethylene glycolbis (succinimidylsuccinate) in PBS for $20 \mathrm{~min}$ at room temperature and then crosslinked with $1 \%$ formaldehyde for $10 \mathrm{~min}$ at room temperature. For ChIP with anti-methylated histone lysine, anti-KLF2 and anti-IRF4, 5-10 $\times 10^{6}$ RPMI8226 cells were crosslinked with $1 \%$ formaldehyde for $10 \mathrm{~min}$ at room temperature. After quenching the reaction with glycine solution, samples were washed twice with ice-cold PBS, resuspended and incubated with micrococcal nuclease for $20 \mathrm{~min}$ at $37^{\circ} \mathrm{C}$. After stopping DNA digestion by adding $50 \mathrm{mM}$ EDTA, nuclear pellets were resuspended and sonicated to break nuclear membranes using Bioruptor (Diagenode) at power level $H$ for $15 \times 30$-s pulses (30-s pause between pulses). After clarifying lysates by centrifugation, supernatants were incubated overnight at $4{ }^{\circ} \mathrm{C}$ with antibodies described in Supplementary Table 1, followed by incubation with Protein $\mathrm{G}$ agarose beads for $2 \mathrm{~h}$. Beads were washed four times, and immune complexes were eluted by heating at $65^{\circ} \mathrm{C}$, with occasional vortexing. Crosslinking was then reversed by incubation overnight at $65^{\circ} \mathrm{C}$. After treating with proteinase $\mathrm{K}$, DNAs were purified with purification spin column. The amount of DNA recovered from immunoprecipitates with specific antibodies or control IgG was determined with quantitative PCR using Applied Biosystems 7300 or 7500 Real-Time PCR system, which was performed in triplicate. Relative occupancy values were determined by calculating the ratios of the amount of immunoprecipitated DNA to that of the input sample. For ChIP with antiKDM3A, anti-KLF2 and anti-IRF4, the values were normalized to the values of control IgG, defined as 1.0. For ChIP with anti-methylated histone lysine, data are shown as fold enrichment compared with sh control (shLuc) samples. Specific primers for each amplified region are shown in Supplementary Table 6.

Affymetrix transcriptome analysis. Total RNAs for microarray analyses were extracted from RPMI8226 cells transduced with shRNA targeting KDM3A or KLF2, or control shRNA in biological duplicate with the RNeasy Mini kit (Qiagen). Total RNA $(1 \mu \mathrm{g})$ was processed, and labelled cRNA was hybridized to Human Genome U133 plus 2.0 arrays (Affymetrix), according to the standard Affymetrix protocols. U133 plus 2.0 is a most comprehensive array that contains $>54,000$ probe sets representing $\sim 38,500$ genes. Expression data can be found at http:// www.ncbi.nlm.nih.gov/geo/ under accession number GSE55667.

Quality control and normalization of transcriptome data. The quality of scanned array images was determined on the basis of background values, RNA degradation plot, array intensity distribution plots, MA plots, per cent present calls, scaling factors and $3^{\prime}-5^{\prime}$ ratio of $\beta$-actin and GAPDH using various bioconductor packages for $\mathrm{R}$ (ref. 60). The normalized transcript expression values were generated from high-quality array images using the Robust Multichip Average algorithm in R using the Bioconductor and associated packages. Robust Multichip Average performed background adjustment, quantile normalization and final summarization of oligonucleotides per transcript using the median polish algorithm ${ }^{61}$. To identify the outlier arrays, unsupervised analysis was performed using principal component analysis and hierarchical clustering. The outlier arrays were deleted from further supervised analysis. Transcripts were filtered to include only those with absolute expression $\geq 40$ in at least $10 \%$ of samples to reduce the false-positive results.

Identification of differentially expressed genes. To identify an accurate signature for target gene knockdown, we performed knockdown using two different shRNAs. Initially individual shRNA signatures of differentially expressed transcripts were generated by comparing control versus target-gene shRNA samples for each shRNA. The transcripts with a $90 \%$ lower confidence bound (LCB) of the fold change between the two groups above 1.5 were considered to be differentially expressed ${ }^{62}$. LCB is a stringent estimate of fold change and has been shown to be the better ranking statistic ${ }^{63}$. Further, a consensus gene knockdown signature was generated from individual shRNA signatures by including genes that were consistently up- or downregulated by at least 1.15 LCB in both shRNAs and an average LCB greater than 1.5 .

Gene set enrichment analysis. GSEA was performed using the GSEA-R, a bioconductor implementation of GSEA from Broad Institute ${ }^{64}$. The GSEA analysis was performed to compare transcriptome signature of $K D M 3 A$ knockdown with KLF2 knockdown and vice versa, or transcriptome signature of IRF4 knockdown with KLF2 knockdown. Before GSEA analysis, normalization of expression data and generation of transcriptome signature were performed using an approach described in the previous section. Transcriptome signature of IRF4 knockdown was obtained from previous report ${ }^{29}$. Analysis was run with 1,000 permutations and a classic statistic; NES, normalized enrichment score and nominal $P$ value was measured.

Construction of the promoter reporter gene. pIRF4(1200) is the human IRF4 promoter-luciferase reporter construct that spans positions $-1,200$ to +14 relative to the transcription start site. pIRF4(520), pIRF4(480) and pIRF4(160) contain deletion constructs with the $5^{\prime}$ end noted in parentheses, and the same $3^{\prime}$ end point at +14 . The $5^{\prime}$ flank region of the human IRF4 was amplified with PCR using genomic DNA isolated from RPMI8226 cells as a template. Then, the digested PCR product was cloned into the XhoI-EcoRV sites of pGL4.10[luc2]
(Promega). Base substitution mutants were generated in pIRF4(520) with the QuikChange II Site-Directed Mutagenesis kit (Agilent Technologies), according to the manufacturer's protocol. The details of the primers are shown in Supplementary Tables 3 and Supplementary Table 4.

Luciferase reporter assay. 293T cells were plated at a density of $1.2 \times 10^{5}$ per 24 -well plate on the day before transfection. Cells were transfected with luciferase reporter plasmid $(0.2 \mu \mathrm{g})$, the indicated amount of expression plasmid and pSV- $\beta$-Gal (Promega; $0.1 \mu \mathrm{g}$ ) with TransIT-LT1 Transfection Reagent (Mirus), according to the manufacturer's instructions. The total amount of DNA per well was kept constant by adding the empty vector, which has the same vector backbone of expression plasmid. After $24 \mathrm{~h}$, the cells were washed with PBS and lysed in $100 \mu \mathrm{l}$ of Reporter Lysis Buffer (Promega). The suspension was incubated for $15 \mathrm{~min}$ at room temperature and centrifuged at top speed in a microfuge for $1 \mathrm{~min}$ The supernatant was used for firefly luciferase activity assay. Values are normalized to $\beta$-galactosidase activity ${ }^{65}$.

MM cell adhesion assay. Bone marrow stromal cells were plated at a density of $1 \times 10^{4}$ per well in a 96-well plate 1 day before the adhesion assay. Human fibronectin- or BSA-coated plates were purchased from R\&D Systems. MM cells were prelabelled by incubating in the presence of $1 \mu \mathrm{M}$ calcein AM (Molecular Probes) at $37^{\circ} \mathrm{C}$ for $30 \mathrm{~min}$. After washing with PBS with calcium and magnesium (PBS( +()$)$ twice, labelled cells were resuspended in $\mathrm{PBS}(+)$ at a concentration of $1 \times 10^{6} \mathrm{ml}^{-1}$. Overall, $1 \times 10^{5}$ cells were then incubated in 96 -well plates coated with bone marrow stromal cells, fibronectin or BSA at $37^{\circ} \mathrm{C}$ for $2 \mathrm{~h}$. After measuring the fluorescent intensity of pre-wash samples, wells were washed two to three times with PBS. The fluorescent intensity of adherent cells was then quantified using a fluorescence plate reader (SpectraMax M3, Molecular Devices) at an excitation wavelength of $485 \mathrm{~nm}$ and an emission wavelength of $525 \mathrm{~nm}$. Per cent adhesion was determined by calculating the ratios of the fluorescent intensity of post-wash sample to that of pre-wash sample.

Transwell migration assay. For migration assay, $4 \times 10^{5} \mathrm{MM}$.1S cells suspended in $200 \mu \mathrm{l}$ of RPMI1640 medium with $1 \%$ fetal bovine serum were placed in the upper chambers, and $500 \mu \mathrm{l}$ of RPMI1640 medium with $1 \%$ fetal bovine serum with CXCL12 $(20 \mathrm{nM})$ were placed in the lower chambers of 24-well transwell plates (pore size $8.0 \mu \mathrm{m}$; Costar). After 4 -h incubation at $37^{\circ} \mathrm{C}$, cells migrated to the lower chambers were labelled with calcein AM (Molecular Probes) at $37^{\circ} \mathrm{C}$ for $1 \mathrm{~h}$ and were quantified using a fluorescence plate reader (SpectraMax M3, Molecular Devices), at an excitation wavelength of $485 \mathrm{~nm}$ and emission wavelength of $525 \mathrm{~nm}$. Overall, $4 \times 10^{5}$ MM.1S cells were also labelled and quantified as input. Per cent migration was determined by calculating the ratios of the fluorescent intensity of migrated cells to that of input cells.

MM cell homing assay. MM.1S-Luc-GFP cells stably expressing the corresponding shRNA (shKDM3A, shKLF2, shIRF4 or control shRFP; $15 \times 10^{6}$ viable cells) were injected via tail vein into 300 -cGy-irradiated NOD-SCID male mice (Charles River Laboratories). Twenty-four hours after injection, mice were killed, and the bilateral femurs of each mouse were collected. After crunching femurs, bone marrow cells are sieved through a $70-\mu \mathrm{m}$ filter, and red blood cells were lysed with ACK Lysing Buffer (Lonza). The bone marrow cells were then resuspended in PBS with $2 \%(\mathrm{v} / \mathrm{v})$ fetal bovine serum, and GFP-positive cells were detected with flow cytometry (BD FACSCanto II, BD Biosciences). Data are shown as relative homing efficacy compared with control cells expressing shRFP. Animal studies were performed under a protocol approved by the Dana-Farber Institutional Animal Care and Use Committee.

Statistical analysis. Student's $t$-test or analysis of variance followed by Dunnett's test was used to compare differences between the treated group and relevant control group. A value of $P<0.05$ was considered significant.

\section{References}

1. Greer, E. L. \& Shi, Y. Histone methylation: a dynamic mark in health, disease and inheritance. Nat. Rev. Genet. 13, 343-357 (2012).

2. Black, J. C., Van Rechem, C. \& Whetstine, J. R. Histone lysine methylation dynamics: establishment, regulation, and biological impact. Mol. Cell 48, 491-507 (2012).

3. Dawson, M. A. \& Kouzarides, T. Cancer epigenetics: from mechanism to therapy. Cell 150, 12-27 (2012).

4. Martinez-Garcia, E. et al. The MMSET histone methyl transferase switches global histone methylation and alters gene expression in $\mathrm{t}(4 ; 14)$ multiple myeloma cells. Blood 117, 211-220 (2011).

5. Kuo, A. J. et al. NSD2 links dimethylation of histone $\mathrm{H} 3$ at lysine 36 to oncogenic programming. Mol. Cell 44, 609-620 (2011).

6. van Haaften, G. et al. Somatic mutations of the histone H3K27 demethylase gene UTX in human cancer. Nat. Genet. 41, 521-523 (2009). 
7. Yamane, K. et al. JHDM2A, a JmjC-containing H3K9 demethylase, facilitates transcription activation by androgen receptor. Cell 125, 483-495 (2006).

8. Okada, Y., Scott, G., Ray, M. K., Mishina, Y. \& Zhang, Y. Histone demethylase JHDM2A is critical for Tnp1 and Prm1 transcription and spermatogenesis. Nature 450, 119-123 (2007).

9. Loh, Y. H., Zhang, W., Chen, X., George, J. \& Ng, H. H. Jmjd1a and Jmjd2c histone H3 Lys 9 demethylases regulate self-renewal in embryonic stem cells. Genes Dev. 21, 2545-2557 (2007).

10. Tateishi, K., Okada, Y., Kallin, E. M. \& Zhang, Y. Role of Jhdm2a in regulating metabolic gene expression and obesity resistance. Nature 458, 757-761 (2009).

11. Inagaki, T. et al. Obesity and metabolic syndrome in histone demethylase JHDM2a-deficient mice. Genes Cells 14, 991-1001 (2009).

12. Kuroki, S. et al. Epigenetic regulation of mouse sex determination by the histone demethylase Jmjd1a. Science 341, 1106-1109 (2013).

13. Parrish, J. K., Sechler, M., Winn, R. A. \& Jedlicka, P. The histone demethylase KDM3A is a microRNA-22-regulated tumor promoter in Ewing Sarcoma. Oncogene 34, 257-262 (2013).

14. Osawa, T. et al. Inhibition of histone demethylase JMJD1A improves anti-angiogenic therapy and reduces tumor-associated macrophages. Cancer Res. 73, 3019-3028 (2013).

15. Uemura, M. et al. Jumonji domain containing $1 \mathrm{~A}$ is a novel prognostic marker for colorectal cancer: in vivo identification from hypoxic tumor cells. Clin. Cancer Res. 16, 4636-4646 (2010).

16. Krieg, A. J. et al. Regulation of the histone demethylase JMJD1A by hypoxia-inducible factor 1 alpha enhances hypoxic gene expression and tumor growth. Mol. Cell Biol. 30, 344-353 (2010).

17. Cho, H. S. et al. The JmjC domain-containing histone demethylase KDM3A is a positive regulator of the G1/S transition in cancer cells via transcriptional regulation of the HOXA1 gene. Int. J. Cancer 131, E179-E189 (2012).

18. Kaczynski, J., Cook, T. \& Urrutia, R. Sp1- and Kruppel-like transcription factors. Genome Biol. 4, 206 (2003).

19. Schuh, W., Meister, S., Herrmann, K., Bradl, H. \& Jack, H. M. Transcriptome analysis in primary B lymphoid precursors following induction of the pre-B cell receptor. Mol. Immunol. 45, 362-375 (2008).

20. Glynne, R., Ghandour, G., Rayner, J., Mack, D. H. \& Goodnow, C. C. B-lymphocyte quiescence, tolerance and activation as viewed by global gene expression profiling on microarrays. Immunol. Rev. 176, 216-246 (2000).

21. Hoek, K. L. et al. Follicular B cell trafficking within the spleen actively restricts humoral immune responses. Immunity 33, 254-265 (2010).

22. Hart, G. T., Wang, X., Hogquist, K. A. \& Jameson, S. C. Kruppel-like factor 2 (KLF2) regulates B-cell reactivity, subset differentiation, and trafficking molecule expression. Proc. Natl Acad. Sci. USA 108, 716-721 (2011).

23. Hart, G. T., Peery, S. L., Hamilton, S. E. \& Jameson, S. C. Cutting edge: Kruppel-like factor 2 is required for phenotypic maintenance but not development of B1 B cells. J. Immunol. 189, 3293-3297 (2012).

24. Winkelmann, R. et al. B cell homeostasis and plasma cell homing controlled by Kruppel-like factor 2. Proc. Natl Acad. Sci. USA 108, 710-715 (2011).

25. Mittrucker, H. W. et al. Requirement for the transcription factor LSIRF/IRF4 for mature B and T lymphocyte function. Science 275, 540-543 (1997).

26. Sciammas, R. et al. Graded expression of interferon regulatory factor-4 coordinates isotype switching with plasma cell differentiation. Immunity 25, 225-236 (2006).

27. Klein, U. et al. Transcription factor IRF4 controls plasma cell differentiation and class-switch recombination. Nat. Immunol. 7, 773-782 (2006).

28. Iida, S. et al. Deregulation of MUM1/IRF4 by chromosomal translocation in multiple myeloma. Nat. Genet. 17, 226-230 (1997).

29. Shaffer, A. L. et al. IRF4 addiction in multiple myeloma. Nature 454, 226-231 (2008).

30. Zhan, F. et al. Gene-expression signature of benign monoclonal gammopathy evident in multiple myeloma is linked to good prognosis. Blood 109, 1692-1700 (2007).

31. Gutierrez, N. C. et al. Gene expression profiling of B lymphocytes and plasma cells from Waldenstrom's macroglobulinemia: comparison with expression patterns of the same cell counterparts from chronic lymphocytic leukemia, multiple myeloma and normal individuals. Leukemia 21, 541-549 (2007).

32. Beyer, S., Kristensen, M. M., Jensen, K. S., Johansen, J. V. \& Staller, P. The histone demethylases JMJD1A and JMJD2B are transcriptional targets of hypoxia-inducible factor HIF. J. Biol. Chem. 283, 36542-36552 (2008).

33. Cory, S. \& Adams, J. M. The Bcl 2 family: regulators of the cellular life-or-death switch. Nat. Rev. Cancer 2, 647-656 (2002).

34. O'Connor, B. P. et al. BCMA is essential for the survival of long-lived bone marrow plasma cells. J. Exp. Med. 199, 91-98 (2004).

35. Peperzak, V. et al. Mcl-1 is essential for the survival of plasma cells. Nat. Immunol. 14, 290-297 (2013).

36. Chapman, M. A. et al. Initial genome sequencing and analysis of multiple myeloma. Nature 471, 467-472 (2011).

37. Andrulis, M. et al. Targeting the BRAF V600E mutation in multiple myeloma. Cancer Discov. 3, 862-869 (2013).
38. Saito, Y. D., Jensen, A. R., Salgia, R. \& Posadas, E. M. Fyn: a novel molecular target in cancer. Cancer 116, 1629-1637 (2010).

39. Sanda, T. et al. Core transcriptional regulatory circuit controlled by the TAL1 complex in human $\mathrm{T}$ cell acute lymphoblastic leukemia. Cancer Cell 22, 209-221 (2012).

40. Boyer, L. A. et al. Core transcriptional regulatory circuitry in human embryonic stem cells. Cell 122, 947-956 (2005).

41. Hideshima, T., Mitsiades, C., Tonon, G., Richardson, P. G. \& Anderson, K. C. Understanding multiple myeloma pathogenesis in the bone marrow to identify new therapeutic targets. Nat. Rev. Cancer 7, 585-598 (2007).

42. McMillin, D. W. et al. Tumor cell-specific bioluminescence platform to identify stroma-induced changes to anticancer drug activity. Nat. Med. 16, 483-489 (2010).

43. Neri, P. et al. Integrin beta7-mediated regulation of multiple myeloma cell adhesion, migration, and invasion. Blood 117, 6202-6213 (2011).

44. Hurt, E. M. et al. Overexpression of c-maf is a frequent oncogenic event in multiple myeloma that promotes proliferation and pathological interactions with bone marrow stroma. Cancer Cell 5, 191-199 (2004).

45. Lohr, J. G. et al. Widespread genetic heterogeneity in multiple myeloma: implications for targeted therapy. Cancer Cell 25, 91-101 (2014).

46. Bolli, N. et al. Heterogeneity of genomic evolution and mutational profiles in multiple myeloma. Nat. Commun. 5, 2997 (2014).

47. Luo, J., Solimini, N. L. \& Elledge, S. J. Principles of cancer therapy: oncogene and non-oncogene addiction. Cell 136, 823-837 (2009).

48. Morito, N. et al. A novel transgenic mouse model of the human multiple myeloma chromosomal translocation $\mathrm{t}(14 ; 16)(\mathrm{q} 32 ; \mathrm{q} 23)$. Cancer Res. 71, 339-348 (2011).

49. Vicente-Duenas, C. et al. A novel molecular mechanism involved in multiple myeloma development revealed by targeting MafB to haematopoietic progenitors. EMBO J. 31, 3704-3717 (2012).

50. Zhan, F. et al. The molecular classification of multiple myeloma. Blood 108, 2020-2028 (2006).

51. Goda, S., Isagawa, T., Chikaoka, Y., Kawamura, T. \& Aburatani, H. Control of histone $\mathrm{H} 3$ Lysine 9 (H3K9) methylation state via cooperative two-step demethylation by Jumonji domain containing 1A (JMJD1A) homodimer. J. Biol. Chem. 288, 36948-36956 (2013).

52. Horton, J. R. et al. Enzymatic and structural insights for substrate specificity of a family of jumonji histone lysine demethylases. Nat. Struct. Mol. Biol. 17, 38-43 (2010).

53. Iida, S. et al. The $\mathrm{t}(9 ; 14)(\mathrm{p} 13 ; \mathrm{q} 32)$ chromosomal translocation associated with lymphoplasmacytoid lymphoma involves the PAX-5 gene. Blood 88, 4110-4117 (1996).

54. Ohno, H. et al. Molecular analysis of a chromosomal translocation, $\mathrm{t}(9 ; 14)(\mathrm{p} 13 ; \mathrm{q} 32)$, in a diffuse large-cell lymphoma cell line expressing the Ki-1 antigen. Proc. Natl Acad. Sci. USA 87, 628-632 (1990).

55. Mimura, N. et al. Blockade of XBP1 splicing by inhibition of IRE1alpha is a promising therapeutic option in multiple myeloma. Blood 119, 5772-5781 (2012).

56. Barretina, J. et al. The Cancer Cell Line Encyclopedia enables predictive modelling of anticancer drug sensitivity. Nature 483, 603-607 (2012).

57. Riising, E. M. et al. Gene silencing triggers polycomb repressive complex 2 recruitment to CpG islands genome wide. Mol. Cell 55, 347-360 (2014).

58. Hideshima, T. et al. NF-kappa B as a therapeutic target in multiple myeloma. J. Biol. Chem. 277, 16639-16647 (2002).

59. Zhang, C., Ao, Z., Seth, A. \& Schlossman, S. F. A mitochondrial membrane protein defined by a novel monoclonal antibody is preferentially detected in apoptotic cells. J. Immunol. 157, 3980-3987 (1996).

60. Kauffmann, A., Gentleman, R. \& Huber, W. arrayQualityMetrics--a bioconductor package for quality assessment of microarray data. Bioinformatics 25, 415-416 (2009).

61. Irizarry, R. A. et al. Exploration, normalization, and summaries of high density oligonucleotide array probe level data. Biostatistics 4, 249-264 (2003).

62. Yuen, T., Wurmbach, E., Pfeffer, R. L., Ebersole, B. J. \& Sealfon, S. C. Accuracy and calibration of commercial oligonucleotide and custom cDNA microarrays. Nucleic Acids Res. 30, e48 (2002).

63. Li, C. \& Wong, W. H. Model-based analysis of oligonucleotide arrays: expression index computation and outlier detection. Proc. Natl Acad. Sci. USA 98, 31-36 (2001).

64. Subramanian, A. et al. Gene set enrichment analysis: a knowledge-based approach for interpreting genome-wide expression profiles. Proc. Natl Acad. Sci. USA 102, 15545-15550 (2005).

65. Ohguchi, H. et al. Hepatocyte nuclear factor 4alpha contributes to thyroid hormone homeostasis by cooperatively regulating the type 1 iodothyronine deiodinase gene with GATA4 and Kruppel-like transcription factor 9. Mol. Cell Biol. 28, 3917-3931 (2008).

\section{Acknowledgements}

We thank Dr James E. Bradner for helpful suggestions and discussions, Dr Takashi Minami for providing the KLF2 antibody (N2212), Drs Bjoern Chapuy and Margaret A. Shipp for providing HBL-1 and SU-DHL4 cell lines and Dr Shinsuke Iida for providing 
KIS-1 cell line. This research was supported by NIH grants SPORE P50 100707 (N.C.M. and K.C.A.), PO-1 78378 (N.C.M. and K.C.A.), PO-1 155258 (M.K.S., N.C.M., and K.C.A.), and RO-1 50947 (K.C.A.). K.C.A. is an American Cancer Society Clinical Research Professor. H.O. was supported in part by a fellowship from the Uehara Memorial Foundation.

\section{Author contributions}

H.O. designed and performed experiments. M.K.B. implemented computational data analysis. L.S. assisted with MM cell homing assay. H.O., T.H., M.K.B., G.T.G., M.C., M.K.S., N.M., R.S., R.D.C., N.R., H.H., T.S., J.S. and K.C.A. analysed the data. Y.-T.T., P.G.R., N.C.M. and K.C.A. provided clinical samples. T.H. and K.C.A. supervised the study. H.O., T.H., M.K.B. and K.C.A. wrote the manuscript.

\section{Additional information}

Accession codes: The microarray data have been deposited in the Gene Expression Omnibus database under accession code GSE55667.
Supplementary Information accompanies this paper at http://www.nature.com/ naturecommunications

Competing financial interests: The authors declare no competing financial interests.

Reprints and permission information is available online at http://npg.nature.com/ reprintsandpermissions/

How to cite this article: Ohguchi, H. et al. The KDM3A-KLF2-IRF4 axis maintains myeloma cell survival. Nat. Commun. 7:10258 doi: 10.1038/ncomms10258 (2016).

\section{(c) (i)}

This work is licensed under a Creative Commons Attribution 4.0 International License. The images or other third party material in this article are included in the article's Creative Commons license, unless indicated otherwise in the credit line; if the material is not included under the Creative Commons license, users will need to obtain permission from the license holder to reproduce the material. To view a copy of this license, visit http://creativecommons.org/licenses/by/4.0/ 